\title{
Modeling Neurodevelopmental Disorders and Epilepsy Caused by Loss of Function of kif2a in Zebrafish
}

\author{
Michèle Partoens, ${ }^{1}$ (D)Ann-Sofie De Meulemeester, ${ }^{1}$ Hoi-Khoanh Giong, ${ }^{2,3,4}$ Duc-Hung Pham, ${ }^{1}$ \\ Jeong-Soo Lee, ${ }^{2,3,4}$ Peter A. de Witte, ${ }^{1}$ and ${ }^{-}$Aleksandra Siekierska ${ }^{1}$
}

https://doi.org/10.1523/ENEURO.0055-21.2021

${ }^{1}$ Laboratory for Molecular Biodiscovery, Department of Pharmaceutical and Pharmacological Sciences, KU Leuven, 3000 Leuven, Belgium, ${ }^{2}$ Disease Target Structure Research Centre, Korea Research Institute of Bioscience and Biotechnology, Daejeon 34141, Republic of Korea, ${ }^{3}$ KRIBB School, University of Science and Technology, Daejeon 34141, Republic of Korea, and ${ }^{4}$ Dementia DTC R\&D Convergence Program, Korea Institute of Science and Technology, Seoul 02792, Republic of Korea

\section{Visual Abstract}

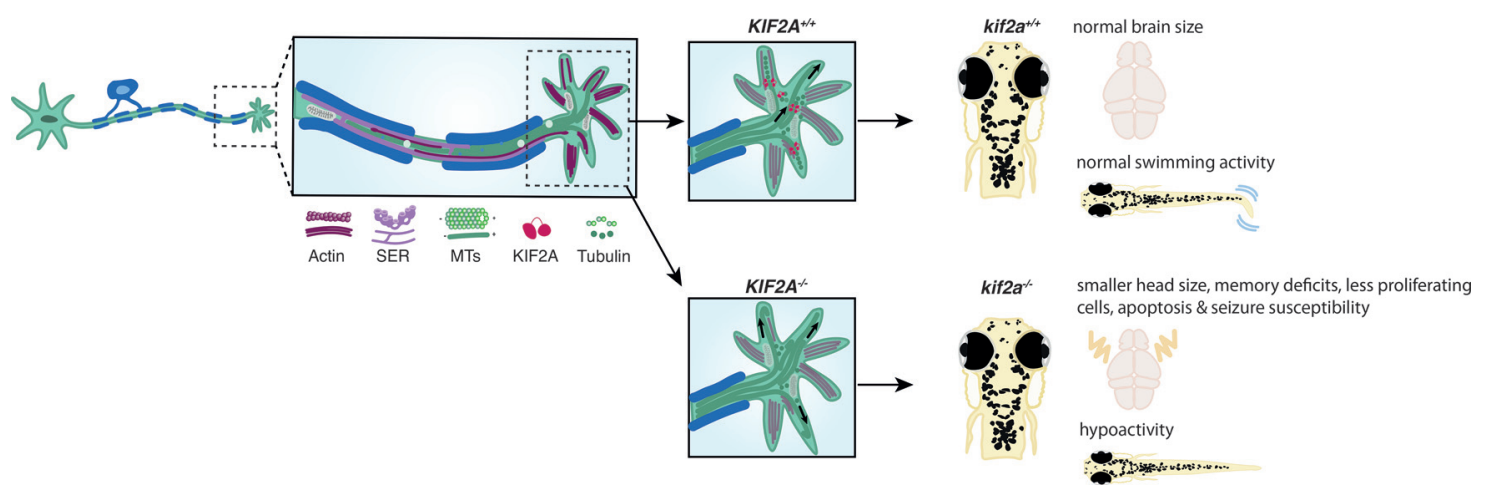

\begin{abstract}
In recent years there has been extensive research on malformations of cortical development (MCDs) that result in clinical features like developmental delay, intellectual disability, and drug-resistant epilepsy (DRE). Various studies highlighted the contribution of microtubule-associated genes (including tubulin and kinesin encoding genes) in MCD development. It has been reported that de novo mutations in KIF2A, a member of the kinesin13 family, are linked to brain malformations and DRE. Although it is known that KIF2A functions by regulating
\end{abstract}

\section{Significance Statement}

This study addresses the functional importance of Kif2a and the phenotypical hallmarks related to KIF2A mutations in zebrafish. We generated a novel kif2a loss-of-function model in zebrafish and demonstrated that Kif2a deficiency was associated with behavioral alterations, habituation deficits, enhanced susceptibility to seizures, microcephaly, neuronal cell proliferation defects, and increased apoptosis. These findings provide insights into a better understanding of the kif2a-related pathologic mechanisms and, eventually, might help in the search for novel and more effective medications to alleviate clinical symptoms typically associated with MCDs. 
microtubule depolymerization via an ATP-driven process, in vivo implications of KIF2A loss of function remain partly unclear. Here, we present a novel kif2a knock-out zebrafish model, showing hypoactivity, habituation deficits, pentylenetetrazole-induced seizure susceptibility and microcephaly, as well as neuronal cell proliferation defects and increased apoptosis. Interestingly, kif2a ${ }^{-1-}$ larvae survived until adulthood and were fertile. Notably, our kif2a zebrafish knock-out model demonstrated many phenotypic similarities to KIF2A mouse models. This study provides valuable insights into the functional importance of kif2a in zebrafish and phenotypical hallmarks related to KIF2A mutations. Ultimately, this model could be used in a future search for more effective therapies that alleviate the clinical symptoms typically associated with MCDs.

Key words: drug-resistant epilepsy; KIF2A; malformations of cortical development; seizures; zebrafish

\section{Introduction}

In humans, proper cortical development is highly dependent on the following three primary time-dependent phases: proliferation of the pool of progenitor cells, along with neuronal migration in the cortical plate, and, ultimately, their differentiation (Diaz and Gleeson, 2009; Sun et al., 2017; Buchsbaum and Cappello, 2019). Alterations in any of these series of orchestrated events may result in a disrupted neuronal circuitry and predispose an individual to the development of neurodevelopmental disorders including malformations of cortical development (MCDs; Leventer et al., 2008; Buchsbaum and Cappello, 2019). MCDs are increasingly recognized as a cause of developmental delay, intellectual disability and drug-resistant epilepsy (DRE), and can be caused by various underlying genetic or exogenous factors (Pang et al., 2008; Buchsbaum and Cappello, 2019). Recent advances in

Received February 9, 2021; accepted August 3, 2021; First published August $17,2021$.

The authors declare no competing financial interests.

Author contributions: M.P., P.A.d.W., and A.S. designed research; M.P., A.-S.D.M., H.-K.G., D.-H.P., and J.-S.L. performed research; M.P., A.-S.D.M., H.-K.G., D.-H.P., J.-S.L., and A.S. analyzed data; M.P., P.A.d.W., and A.S. wrote the paper.

This study received funding from the Scientific Research Foundation Flanders (FWO). M.P. is a PhD fellow of the Fund for Research Foundation Flanders (FWO, Grant $1 \mathrm{~S} 84418 \mathrm{~N}$ ) and A.-S.D.M. is a PhD fellow of the Fund for O6260 Research Foundation Flanders (FWO, Grant 11F2919N). J.-S.L. was supported by the National Research Council of Science \& Technology of the Ministry of Science and ICT of Korea (Grant CRC-15-04-KIST) and the KRIBB Research Initiative Program. A.S. was supported by KU Leuven Internal Funding C3 (Grant C32/18/067). The Cell and Tissue Imaging Cluster at KU Leuven was supported by Hercules Grant AKUL/11/37 and FWO Grant G.0929.15.

D.-H. Pham's present address: Division of Immunobiology, Cincinnati Children's Hospital Medical Center, 3333 Burnet Avenue, Cincinnati, OH 45229.

We thank Dr. Mario F. Wullimann for valuable comments concerning the histological analysis. We also thank the Skeletal Biology and Engineering Research Center (KU Leuven) for using the microtome and automatic staining machine. In particular, we would like to thank An Hens, Lies Storms, and Elena Nefyodova for input regarding histological experiments. In addition, we thank Dr. Wietske van der Ent for assistance with BrdU cell proliferation analysis. Finally, we thank Prof. Pieter Vanden Berghe (KU Leuven) for use of the Zeiss LSM 780-SP Mai Tai HP DS confocal microscope (in the Cell and Tissue Imaging Cluster).

Correspondence should be addressed to Peter A. de Witte at peter. dewitte@kuleuven.be.

https://doi.org/10.1523/ENEURO.0055-21.2021

Copyright $\odot 2021$ Partoens et al.

This is an open-access article distributed under the terms of the Creative Commons Attribution 4.0 International license, which permits unrestricted use, distribution and reproduction in any medium provided that the original work is properly attributed. genetics have led to the discovery of a number of new genes associated with MCDs. Among these are kinesin superfamily genes (KIFs), encoding ATP-driven microtubule-associated proteins that use the energy of ATP hydrolysis to carry out mechanical work along microtubule tracks (Poirier et al., 2013; Ali and Yang, 2020). It has been reported that de novo mutations in kinesin family member 2a (KIF2A) represent a significant cause of lissencephaly, microcephaly, and DRE (Bahi-Buisson and Cavallin, 2016; Homma et al., 2003; Poirier et al., 2013; Guerrini and Dobyns, 2014; Tian et al., 2016; Cavallin et al., 2017; Broix et al., 2018). KIF2A belongs to the human kinesin-13 family (M-kinesins) consisting of KIF2A, KIF2B, KIF2C/MCAK, and KIF24 (Miki et al., 2005), and is highly abundant in developing neurons being involved in neuronal migration, axonal elongation, and pruning (Homma et al., 2003, 2018; Maor-Nof et al., 2013; Broix et al., 2018). Several studies demonstrated that multiple KIF2A mutations found in patients are located in or near the ATP binding site of the kinesin motor domain (residues p.S317, p.T320, and p.H321; Poirier et al., 2013; Guerrini and Dobyns, 2014; Tian et al., 2016; Cavallin et al., 2017), thus affecting ATP hydrolysis or microtubule binding, ultimately resulting in a nonfunctional kinesin (Poirier et al., 2013).

Since epilepsy is the most common clinical consequence of MCDs (Leventer et al., 1999, 2008; Guerrini and Dobyns, 2014; Tian et al., 2016; Cavallin et al., 2017), antiepileptic drugs are used to control seizures; however, many patients remain unresponsive to the medication therapy (Represa, 2019). Hence, there is an unmet medical need to advance our understanding of the pathogenesis of MCDs and related epilepsies. In recent years, mouse KIF2A models have been developed, which has led to important insights concerning cortical defects and epilepsy (Homma et al., 2003, 2018; Gilet et al., 2020). Kif2a-null mice showed multiple neurodevelopmental abnormalities like early postnatal death, cortical lamination defects as a result of aberrant neuronal migration, and lateral cortical ventricle enlargement; however, no manifestation of epileptic seizures could be reported (Homma et al., 2003). Further characterization of the disease phenotype was allowed by a tamoxifen-inducible Kif2a conditional knock-out (KO) mouse model, demonstrating the role of KIF2A in the precise postnatal hippocampal wiring (Homma

Author contributions: M.P., P.A.d.W., and A.S. designed research; M.P., A.-S.D.M., H.-K.G., D.-H.P., and J.-S.L. performed research; M.P., A.-S.D.M., H.-K.G., D.-H.P., J.-S.L., and A.S. analyzed data; M.P., P.A.d.W., and A.S. wrote the paper. 
et al., 2018). Although neuronal proliferation or migration was not disrupted, mice displayed severe epilepsy, likely a cause of death at 6 weeks postbirth (Homma et al., 2018). Recently, a conditional knock-in (KI) mouse model was developed, bearing the human KIF2A p. H321D missense point variant identified in MCD patients (Gilet et al., 2020). KIF2A $A^{+/ H 321 D} \mathrm{KI}$ mice survived and displayed microcephaly and neuroanatomical anomalies like hippocampal structure abnormalities and cortical layer disorganization, resulting from abnormal neuronal migration and increased cell death (Gilet et al., 2020). Moreover, KIF2A-deficient animals showed behavioral deficits and susceptibility to epilepsy, correlating with the described human phenotype.

Over the years, the zebrafish has emerged as a promising model to study neurodevelopmental diseases and their pathologic processes in early development. The developing larval zebrafish brain presents numerous similar structures and cell types that are found in mammals (Kalueff et al., 2014). Moreover, their small size, high fecundity, ex utero development, and genetic amenability allow for higher-throughput screenings, improving CNS drug discovery (Stewart et al., 2015; Khan et al., 2017).

This study intends to advance our understanding of KIF2A-related MCDs by investigating the pathologic consequences of Kif2a loss of function in vivo in a novel zebrafish model. By means of neurobehavioral and electrophysiological assays, we demonstrated that a loss of Kif2a in zebrafish causes behavioral alterations like hypoactivity and habituation deficits, reduced head size, neuronal cell proliferation defects, apoptosis, and, importantly, increased pentylenetetrazole (PTZ)-induced seizure susceptibility. Despite these abnormalities, kif2a ${ }^{-/-}$ larvae survived until adulthood and were fertile. Altogether, our results show that the kif2a KO model could ultimately be used in the search for novel and more effective medications that alleviate the clinical symptoms associated with MCDs.

\section{Materials and Methods}

\section{Zebrafish husbandry}

All zebrafish (Danio rerio) strains used in this study were maintained at $28 \pm 2^{\circ} \mathrm{C}$ on a $14: 10 \mathrm{~h}$ light/dark cycle under standard aquaculture conditions in a UV-sterilized rack recirculating system equipped with a mechanical and biological filtration unit. Embryos were collected via natural spawning and immediately transferred to Danieau's medium ( $0.3 \times$ Danieau's medium), containing 1.5 mM HEPES buffer pH 7.2, $17.4 \mathrm{~mm} \mathrm{NaCl}$, $0.21 \mathrm{~mm} \mathrm{KCl}, 0.18 \mathrm{~mm} \mathrm{Ca}\left(\mathrm{NO}_{3}\right)_{2}, 0.12 \mathrm{~mm} \mathrm{MgSO}$, and $0.6 \mu \mathrm{m}$ methylene blue. For the experiments, embryos and larvae were kept at $28.5^{\circ} \mathrm{C}$ with a photoperiod of $14 \mathrm{~h}$. In all experiments, zebrafish larvae were treated in a humane way and with regard for alleviation of suffering. All zebrafish experiments were performed in accordance with the guidelines of and approval by the Ethical Committee of the University of Leuven (P023/ 2017 and P027/2019) and by the Belgian Federal Department of Public Health, Food Safety and Environment (LA1210199).

\section{Generation of the kif2a CRISPR knock-out zebrafish line}

A kif2a knock-out line was generated via the CRISPR/ Cas9 technique (Hruscha et al., 2013; Hwang et al., 2013). kif2a single-guide RNA (sgRNA) targeting exon 5 in the kif2a gene $\left(5^{\prime}\right.$-CAGCCAGAATCAGCACCCCC- $\left.3^{\prime}\right)$ was designed using the CHOP CHOP web tool (https://chopchop.cbu.uib. no), and was further transcribed using the MEGAshortscript T7 Transcription Kit (Ambion) and purified with the MEGAclear Transcription Clean-Up Kit (Ambion). Cas9 (GeneArt CRISPR Nuclease mRNA) was purchased from Thermo Fisher Scientific. Single cell-stage, fertilized wild-type embryos of the $A B$ line were injected with $100 \mathrm{pg}$ of kif2a sgRNA and $150 \mathrm{pg}$ of Cas 9 mRNA (in $1 \mathrm{nl}$ volume). The mutation at the target site was verified via T7 endonuclease assay. The remaining sgRNA/Cas9injected embryos were raised till adulthood, outcrossed with wild-type adults, and screened for indels by Sanger sequencing. FO founder with germline transmission and a high rate of indels was selected to establish the knock-out line. F1 generation embryos of F0 founder were raised to adulthood, fin clipped, and sequenced. Individuals carrying $4 \mathrm{bp}$ deletion of CCAG were identified and pooled together. Experiments were performed on embryos coming from homozygous and heterozygous F2 or F3 progeny.

To confirm the genotype of the larvae, a fin clip was placed in separate tubes with $50 \mu \mathrm{l}$ of lysis buffer $(100 \mu \mathrm{M}$ Tris, $10 \mu \mathrm{m}$ EDTA, $0.7 \mathrm{~mm}$ proteinase $\mathrm{K}, 0.2 \%$ Triton $\mathrm{X}-100$ in Milli-Q water) to extract genomic DNA. Lysis was performed at $55^{\circ} \mathrm{C}$ for $3 \mathrm{~h}$, followed by $10 \mathrm{~min}$ at $95^{\circ} \mathrm{C}$. Lysed samples were genotyped by performing a PCR to amplify the genomic region spanning $4 \mathrm{bp}$ deletion using KAPA HiFi HotStart Polymerase (Roche) and kif2a-specific primers. Successfully amplified PCR products were purified using QIAquick PCR Purification Kit (Qiagen), and the sequence was verified with Sanger sequencing (LGC Genomics). The genotypes of the individual larvae were analyzed using SeqMan software (DNASTAR Lasergene).

\section{Survival assay}

From 1 to $5 \mathrm{~d}$ postfertilization (dpf) zebrafish embryos were cultured in Danieau's medium in a $10 \mathrm{~cm}$ Petri dish. At $6 \mathrm{dpf}$, they were transferred to a specialized infant incubator system at $28.5^{\circ} \mathrm{C}$ on a $14: 10 \mathrm{~h}$ light/dark cycle. They were fed three times a day with $1 \mathrm{dpf}$ brine shrimp and dry granular food (SDS). Larvae were checked every day to a juvenile stage (30 dpf). Dead or moribund zebrafish larvae were registered, removed from the system, and frozen until further analysis. After a period of $30 \mathrm{~d}$, larvae were genotyped as described previously, and survival curves were generated using a Kaplan-Meier estimate.

\section{Whole-mount RNA in situ hybridization}

The $973 \mathrm{bp}$ coding sequence fragment of kif2a was amplified from cDNA of $A B$ wild-type strain and cloned into a Zero Blunt TOPO PCR Cloning Kit (Thermo Fisher Scientific). Cloned DNA was linearized by Xhol and HindIII, then synthesized by SP6 RNA polymerase and 
T7 RNA polymerase using DIG RNA labeling kit (all Roche) for sense- and antisense DIG-labeled RNA probes, respectively.

Embryos were fixed in 4\% PFA, then washed with $1 \times$ PBS with Tween $20(1 \times$ PBST), sequentially washed with $100-25 \%$ methanol and stored in $100 \%$ methanol at $-20^{\circ}$ $\mathrm{C}$ until needed. On the first day of whole-mount RNA in situ hybridization, embryos were washed with 50-25\% methanol, followed by $1 \times$ PBST. After treatment with proteinase K (Sigma-Aldrich) according to the developing stages for permeabilization, embryos were fixed again with 4\% PFA and washed by $1 \times$ PBST. Embryos were hybridized in $\mathrm{Hyb}^{+}$solution with the kif2a RNA probes at $70^{\circ} \mathrm{C}$ overnight. On the second day, after serial washing with $2 \times$ SSCT (sodium citrate buffer with $0.1 \%$ Tween 20 ) with $50 \%$ formamide, $2 \times \mathrm{SSCT}$, and $0.2 \times \mathrm{SSCT}$ at $70^{\circ} \mathrm{C}$, embryos were blocked with $5 \%$ horse serum (SigmaAldrich) and incubated with anti-digoxigenin-AP Fab fragments (Roche) overnight at $4^{\circ} \mathrm{C}$. On the third day, embryos were developed with BCIP/NBT (nitroblue tetrazolium) substrate (Roche). Staining was developed and stopped before the background signals started to appear in the embryos hybridized with the sense RNA probe.

\section{RNA extraction and quantitative RT-PCR analysis}

Total RNA was extracted using TRIzol reagent (Thermo Fisher Scientific), followed by phenol-chloroform extraction, isopropanol precipitation, and ethanol washes. The resulting RNA pellet was air dried, dissolved in nuclease-free water (Thermo Fisher Scientific), and subsequently treated with RNase-free DNase (Roche). Then, $1 \mu \mathrm{g}$ of total RNA was reverse transcribed with the High Capacity cDNA Reverse Transcription Kit (Thermo Fisher Scientific) according to the manufacturer protocol. Next, the generated cDNA was diluted (1:20) and amplified using kif2a- (forward, 5'-GATCACTATTCCAAGTAAA-3'; reverse, 5'-CCACTTCCTGTTTGACCATA-3') and kif2c-specific primers (forward, 5' -CAAGAAGAATGACCACGCGT-3'; reverse, 5'-AGTCTCCTCTGGTAGCCTGA-3') and 2× SsoAdvanced Universal SYBR Green Supermix (Bio-Rad) in Hard-Shell Low Profile Thin-Wall 96-well skirted PCR plates (Bio-Rad) on a CFX96 touch RT-PCR detection system (Bio-Rad) under cycling conditions according to the manufacturer protocol. The relative expression levels were quantified using the comparative Cq method ( $\Delta \Delta \mathrm{Cq}$ ) with CFX Maestro software (Bio-Rad). kif2a and kif2c transcripts were normalized against $\beta$-actin using specific primers (forward, 5'-TACAATGAGCTCCGTG TTGC-3'; reverse, 5'-TACAATGAGCTCCGTGTTGC-3'; and forward, 5'CAACAACCTGCTGGGCAAA-3'; reverse, 5' GCGTCGATGTCGAAGGTCA-3', respectively).

\section{Western blotting}

Zebrafish larvae of 1-7 dpf were pooled (15 embryos for $1 \mathrm{dpf}$ and 10 larvae for 2-7 dpf; all viable at the time of collection) and lysed in RIPA buffer (Sigma-Aldrich). Following the BCA assay, $30 \mu \mathrm{g}$ of total protein was loaded onto 4$12 \%$ Bis-Tris mini gels (Thermo Fisher Scientific), separated by electrophoresis according to their molecular weight and electroblotted on nitrocellulose membranes
(Thermo Fisher Scientific). Membranes were blocked in Intercept blocking buffer (LI-COR) for an hour at room temperature $(R T)$, then incubated overnight with anti-KIF2A polyclonal rabbit antibody (catalog \# ab71160, Abcam) or anti-GAPDH polyclonal antibody (catalog \#SAB2701826, Sigma-Aldrich) at $4^{\circ} \mathrm{C}$ and subsequently with a DyLight $8004 \times$ polyethylene glycol-conjugated secondary antibody (catalog \#SA5-35571, Thermo Fisher Scientific) for $1 \mathrm{~h}$ at RT. Afterward, membranes were washed three times with $1 \times$ TBST for $15 \mathrm{~min}$. The proteins were visualized by a biomolecular imaging system (Typhoon NIR, GE Healthcare) and bands quantified with Image Studio Lite software.

\section{Behavioral studies}

Locomotor studies were performed as published previously (Scheldeman et al., 2017; Siekierska et al., 2019). Five to eight days postfertilization zebrafish larvae were individually arrayed in a 24-well plate containing $400 \mu \mathrm{l}$ of Danieau's medium per well, followed by a 20 min habituation period in an automated tracking device (Zebrabox, Viewpoint). The average total locomotor activity was quantified during a 5 min light and 10 min dark period using ZebraLab software (Viewpoint) and expressed in actinteg units, defined as the sum of all image pixel changes detected during the time of the tracking experiment. Every day, before the experiment, larvae were replenished with $400 \mu \mathrm{l}$ of fresh Danieau's medium. The results consist of data from quadruple experiments.

PTZ experiments were executed as published previously (Afrikanova et al., 2013). Larvae were individually arrayed in a 24-well plate containing $400 \mu$ of Danieau's medium per well. Subsequently, an equal volume of 12 mм PTZ (Sigma-Aldrich) was added before recording to obtain a $6 \mathrm{~mm}$ final PTZ concentration. The average total locomotor activity was quantified during a 30 min period using ZebraLab software (Viewpoint) and expressed in actinteg units. The results consist of data from quadruple experiments.

For habituation assay, a previously published protocol was adapted (Wolman et al., 2011). Six days postfertilization larvae were individually arrayed in a 96-well plate containing $100 \mu$ l of Danieau's medium per well and equilibrated for $3 \mathrm{~h}$ in the uniformly illuminated automated tracking device (Zebrabox, Viewpoint). Subsequently, the larvae were subjected to a spaced training consisting of 120 dark flashes (DFs) with $15 \mathrm{~s}$ interstimulus intervals (ISIs) alternated with 10 min light periods. After a 30 min light pause, the larvae were exposed to the test assay consisting of 10 DFs with $60 \mathrm{~s}$ ISIs. Total movement was expressed in actinteg units. The analysis of the habituation assay was performed as previously described by Nelson et al. (2020). Spaced training results were presented as a percentage of habituation using a formula 1 (average movement from last 30 DFs/average movement from first $30 \mathrm{DFs}$ ) * 100 per larva. Test results were presented as a habituation ratio of the average movement between 10 DFs from the test and the last 10 DFs from the spaced training. The results consist of data from three experiments. 


\section{Electrophysiological studies}

Noninvasive local field recordings were performed as published previously (Scheldeman et al., 2017; Siekierska et al., 2019). A 5 dpf larva was immobilized in $2 \%$ lowmelting point agarose (Thermo Fisher Scientific), and the electric signal was recorded using a glass electrode that was placed on the skin of the head of a larva above the optic tectum, connected to a high-impedance amplifier filled with artificial CSF (124 mm NaCl, $2 \mathrm{~mm} \mathrm{KCl,} 2 \mathrm{~mm} \mathrm{MgSO}_{4} 2 \mathrm{~mm} \mathrm{CaCl}_{2}$, $1.25 \mathrm{~mm} \mathrm{K \textrm {K } _ { 2 } \mathrm { PO } _ { 4 } , 2 6 \mathrm { mm } \mathrm { NaHCO }}$, and $10 \mathrm{~mm}$ glucose). A grounding electrode and a reference electrode were placed in the recording space. The differential signal between the recording electrode and the reference electrode was amplified 10,000 times by a DAGAN 2400 Amplifier, band pass filtered at $0.3-300 \mathrm{~Hz}$, and digitized at $2 \mathrm{kHz}$ via a PCl-6251 interface (National Instruments) with WinEDR (John Dempster, University of Strathclyde, Glasgow, UK). Single recordings were performed for $10 \mathrm{~min}$. Spontaneous electrical discharges were classified as positive events when their amplitude was at least three times the amplitude of the baseline and lasted for at least $100 \mathrm{~ms}$. Analysis of the recordings was performed in a blinded manner using Clampfit 10.2 software (Molecular Devices).

PTZ experiments were executed as described previously (Afrikanova et al., 2013), larvae were arrayed in a 96-well plate containing $100 \mu \mathrm{l}$ of Danieau's medium. Subsequently, an equal volume of $12 \mathrm{~mm}$ PTZ (SigmaAldrich) was added for $15 \mathrm{~min}$ before recording to obtain a $6 \mathrm{~mm}$ final PTZ concentration. Electrophysiological recordings were performed and analyzed as described above. Data were expressed as the mean \pm SD.

\section{Head and body surface area measurements}

For head area measurements, larvae were positioned in $3 \%$ methylcellulose. Lateral images of 3 and $5 \mathrm{dpf}$ larvae were acquired with a microscope (model MZ 10F, Leica; with a DFC310 FX digital camera, Leica) and Leica Application Suite version 3.6 software. Measurements were performed manually in ImageJ software in a blind manner. Zebrafish body surface area was measured by encircling the total surface of a larva, starting from the anterior tip of the snout to the base of the posterior caudal fin. Head surface areas were measured by tracing the boundary of the surface of interest using reference spots on the head such as the otic vesicle or dorsal indentation just above the eye, at the level of the pineal gland, as previously published (Scheldeman et al., 2017; Siekierska et al., 2019). The absolute values of measured head surface area were normalized to the body surface area of the larvae.

\section{Hematoxylin and eosin staining}

The $5 \mathrm{dpf}$ larvae were fixed in $4 \%$ PFA at $4^{\circ} \mathrm{C}$ overnight and kept in $70 \%$ ethanol. At least four larvae per genotype group were embedded in $1 \%$ agarose in $1 \times$ TAE buffer. A mold, specifically designed to align zebrafish larvae, was used to produce agarose blocks with identical distributed wells of the same depth. Agarose blocks were gradually dehydrated in an enclosed automated tissue processor
(Shandon Excelsior ES, Thermo Fisher Scientific) and subsequently embedded in paraffin. The heads of paraffin-embedded larvae were sectioned on an HM 325 manual rotary microtome (Thermo Fisher Scientific) at a thickness of $5 \mu \mathrm{m}$. The specimens were stained with hematoxylin and eosin (H\&E) stain using Varistain Gemini ES Automated Slide Stainer (Thermo Fisher Scientific) according to laboratory protocols. The resulting sections were imaged at $20 \times$ and $40 \times$ magnification in SPOT 5.1 software (SPOT Imaging) by a SPOT-RT3 camera mounted on a Leica microscope. The brightness of the images was adjusted for the white background. The number of sections was counted per larva per genotype and expressed as the mean \pm SD. Additional counting of brain nuclei was performed to support size difference. Four equivalent sections were selected for each brain region per genotype, and hematoxylin-positive stained nuclei were counted using QuPath (0.2.3) software. The results were expressed as the average of hematoxylinpositive stained nuclei within each selected brain area.

\section{Whole-mount BrdU labeling}

Whole-mount BrdU staining was performed on $5 \mathrm{dpf}$ larvae as described previously (Tiraboschi et al., 2020). Larvae were incubated in a $10 \mathrm{~mm}$ BrdU (Sigma-Aldrich): $1 \times$ E3 with $15 \%$ DMSO solution for $20 \mathrm{~min}$ on ice. Embryos were placed at $28^{\circ} \mathrm{C}$ for $5 \mathrm{~min}$ and subsequently fixed for $2 \mathrm{~h}$ in $4 \%$ PFA at RT. Fixed larvae were washed three times for $5 \mathrm{~min}$ in $0.1 \%$ PBS-Tween at RT, incubated in $150 \mathrm{~mm}$ Tris- $\mathrm{HCl}, \mathrm{pH}$ 9, for $15 \mathrm{~min}$ at $\mathrm{RT}$, then at $70^{\circ} \mathrm{C}$ for $15 \mathrm{~min}$. Larvae were washed three times for $5 \mathrm{~min}$ in $0.1 \%$ PBS-Tween and permeabilized in acetone for $30 \mathrm{~min}$ at $-20^{\circ} \mathrm{C}$. Afterward, larvae were incubated in $2 \mathrm{~N}$ $\mathrm{HCl}$ for $1 \mathrm{~h}$ at $\mathrm{RT}$ to expose the BrdU epitope. Next, $\mathrm{HCl}$ was neutralized with $0.1 \mathrm{M}$ borate buffer, $\mathrm{pH} 8.5$, for $20 \mathrm{~min}$ at RT, after which larvae were washed $3 \times$ for $5 \mathrm{~min}$ in $0.1 \%$ PBS-Tween and afterward, again for $15 \mathrm{~min}$. Larvae were bathed overnight in blocking buffer at $4^{\circ} \mathrm{C}$ in $0.1 \%$ PBS-Tween $/ 10 \%$ goat serum $/ 0.8 \%$ Triton $X-$ $100 / 1 \%$ BSA on a rotary shaker. The day after, blocking buffer was exchanged for BrdU antibody (clone 3D4; dilution, 1:200; BD Biosciences) in 0.1\% PBSTween $1 \%$ goat serum $/ 0.8 \%$ Triton $\mathrm{X}-100 / 1 \%$ BSA for $72 \mathrm{~h}$ at $4^{\circ} \mathrm{C}$. Further, larvae were washed $3 \times$ for $5 \mathrm{~min}$ in $0.1 \%$ PBS-Tween and incubated for $5 \mathrm{~h}$ in $0.1 \%$ PBS-Tween/0.8\% Triton X-100. Afterward, secondary antibody (dilution, 1:2000; Alexa Fluor 594 goat-antimouse, Abcam) and NucRed (dilution, 1:5000; Live 647 ReadyProbes Reagent, Thermo Fisher Scientific) were applied in $0.1 \%$ PBS-Tween $/ 1 \%$ goat serum/ $0.8 \%$ Triton $\mathrm{X}-100 / 1 \% \mathrm{BSA}$ for $72 \mathrm{~h}$ at $4^{\circ} \mathrm{C}$. After $72 \mathrm{~h}$, larvae were washed $3 \times$ for 5 min in $0.1 \%$ PBS-Tween and incubated overnight in $0.1 \%$ PBS-Tween $/ 0.8 \%$ Triton X-100. The day after, larvae were mounted on glass slides and dorsal $z$-stacks of the optic tectum were acquired using an LSM 780-SP Mai Tai HP DS confocal microscope (Zeiss) equipped with an LD LCI Plan Apo $25 \times / 0.8$ objective. To quantify proliferative cells in the optic tectum using ImageJ, a nonspecific signal was removed by creating a mask on the nuclear dye channel. Then, a threshold was set in the BrdU 
channel to identify individual proliferative cells, and particle analysis was performed on a z-projection of this signal.

\section{Caspase-3 immunohistochemistry}

Immunohistochemical detection of cell death was conducted on 5- $\mu \mathrm{m}$-thick deparaffinized and rehydrated sections corresponding to the sections stained with H\&E, as published previously (Siekierska et al., 2019). Specimens were subjected to heat-induced antigen retrieval by incubation in $10 \mathrm{~mm}$ sodium citrate, $\mathrm{pH}$ 6.0 , for $10 \mathrm{~min}$ at $98^{\circ} \mathrm{C}$ in a water bath, followed by a 30 min cool down. Subsequently, specimens were treated twice for $8 \mathrm{~min}$ with $3 \%$ hydrogen peroxide. The sections were blocked for 30 min in $5 \%$ normal goat serum in $1 \times$ TBST at RT, and further incubated with primary antibody against active caspase-3 (1:500 dilution; catalog \#ab13847, Abcam) for $1 \mathrm{~h}$ at RT. After rinsing $3 \times$ for 15 min with $1 \times$ TBST, an HRP-conjugated secondary antibody (1:200 dilution; catalog \#111-035-003, Jackson ImmunoResearch) was applied for $1 \mathrm{~h}$ at RT. The slides were treated with $\mathrm{DAB}^{+}$/chromogen (DAKO) for $1 \mathrm{~min}$ at RT and rinsed with deionized water. As a nuclear counterstain, hematoxylin was added for $3 \mathrm{~min}$ and the specimens were subsequently rinsed in deionized water. After clearing in ethanol and histoclear, the slides were mounted. For each staining, a negative control was included by processing sections in the absence of the primary antibody. The images were taken at $20 \times$ magnification in a SPOT 5.1 software (SPOT Imaging) by a SPOT-RT3 camera mounted on a Leica microscope. The brightness of the images was adjusted for the white background. The number of DAB positively stained nuclei in the whole brain was counted using QuPath (0.2.3) software per larva per genotype. The results were expressed as the percentage of apoptotic cells $\pm S D$ (DAB positively stained nuclei over the total cell number) per larva per genotype.

\section{Experimental design and statistical analysis}

When comparing parametric data from two groups, an unpaired Student's $t$ test was used. If the data were from more than two groups, a one-way ANOVA with a Dunnett's multiple-comparisons test were used (Table 1). Outliers were identified from datasets using the ROUT method $(Q=1 \%)$. The significance of all statistical comparisons was set at $p<0.05$. All statistical comparisons were performed using GraphPad Prism (version 8.0.0) software.

\section{Results}

\section{Mutation of the kif2a gene by CRISPR/Cas9 results in a stable homozygous mutant zebrafish line}

Zebrafish genome encodes a single KIF2A ortholog that shows a high degree of conservation between mouse and human proteins (specifically within the kinesin motor domain), with $84.66 \%$ identity and $89 \%$ similarity for human and zebrafish (Fig. 1a). To investigate KIF2A loss of function in vivo, a zebrafish knock-out model was generated by the CRISPR/Cas9 genome-editing technology (Hruscha et al., 2013; Hwang et al., 2013). We targeted the fifth exon of the zebrafish kif2a located in the globular $\mathrm{N}$-terminal domain. A positive founder, transmitting a premature stop codon, was ultimately selected and used to obtain the F2 generation (Extended Data Fig. 1-1a,b). Genomic analysis confirmed the presence of the mutation in kif2a $a^{-1-}$ larvae (Fig. 1b).

\section{$\mathrm{kif2a}^{-/-}$larvae survive until adulthood and are fertile}

Homozygous larvae obtained by the mating of heterozygous adults segregated according to a Mendelian ratio $(1: 2: 1)$. Surprisingly, kif2a $^{-1-}$ larvae were able to survive until adulthood. Kaplan-Meier curves showed that at 30 dpf the recorded survival rate was $75 \%$ for $k^{2} 2 a^{+/+}$ $(n=5), 60 \%$ for $\mathrm{kif2a}^{-/-}(n=10)$, and $57 \%$ for $\mathrm{kif}_{2} \mathrm{a}^{+/-}$ $(n=21)$ larvae $\left(\chi^{2}=0.65, p=0.72\right.$, log-rank Mantel-Cox test; Fig. 1c). Moreover, the offspring of incrossed homozygotes was viable and able to be raised until adulthood, and these homozygous progenies were used to perform the experiments described in this study, unless differently specified.

Visual inspection of the development of $\mathrm{kif2a}^{+/+}$and

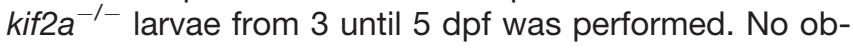
vious macroscopic differences in appearance such as gross deformation, edema, necrosis, or impairment in touch response were detected, making $\mathrm{kif2}^{-/-}$larvae morphologically inconspicuous when compared with $k_{i f 2 a^{+/+}}$animals (Fig. 1d). The same trend was observed for adult mutants, which were all morphologically similar to their $\mathrm{kif}_{2} \mathrm{a}^{+/+}$and $\mathrm{kif2}^{+\mathrm{a}^{+/-}}$siblings (data not shown).

Since $\mathrm{kif}^{2} \mathrm{a}^{-1-}$ larvae were viable and morphologically indistinguishable from their kif2a ${ }^{+/+}$siblings, we investigated whether the loss of Kif2a might result in a direct genetic compensation from another member of kinesin-13 family, kif2c. Quantitative PCR (qPCR) results did not show any increase in kif2c expression levels in kif2a $a^{-1-}$ larvae compared with their $k i f 2 a^{+/+}$siblings either at $2 \mathrm{dpf}$ $\left(k_{i f 2 a}{ }^{-/-}: 1.142 \pm 0.3583, n=5 ;\right.$ kif $^{+/+}: 1.0 \pm 0.0, n=5$, Student's $t$ test; Extended Data Fig. 1-1c) or at $5 \mathrm{dpf}$ $\left(k_{i f 2 a} a^{-l-}: 1.098 \pm 0.2887, n=2 ; k_{i f 2 a}{ }^{+/+}: 1.0 \pm 0.0, n=2\right.$, Student's $t$ test; Extended Data Fig. 1-1d; i.e., at time points of peak and declining levels of kif2a RNA and Kif2a protein expression, respectively) observed in $k i f 2 a^{+/+}$ larvae.

\section{Temporal and spatial expression patterns reveal a loss of kif2a mRNA in the mutant line}

We investigated the expression levels of kif2a RNA and Kif2a protein during 1-7 dpf. Both kif2a transcript (Fig. 2a) and Kif2a protein (Fig. 2b) expression were found to increase during the first days of development, with peak expression levels at $2 \mathrm{dpf}$ (transcript: $2119 \pm 238.2, n=3$; protein: $1.609 \pm 0.1993, n=3$ ) and $3 \mathrm{dpf}$ (transcript: $2077 \pm 259.8, n=3$; protein: $1.459 \pm 0.2164, n=3$ ). At 5 dpf (transcript: $418.8 \pm 57.25, n=3$; protein: $0.3680 \pm$ $0.2259, n=3)$, however, the expression decreased, with an almost complete absence of protein at $7 \mathrm{dpf}(0.1299 \pm$ 

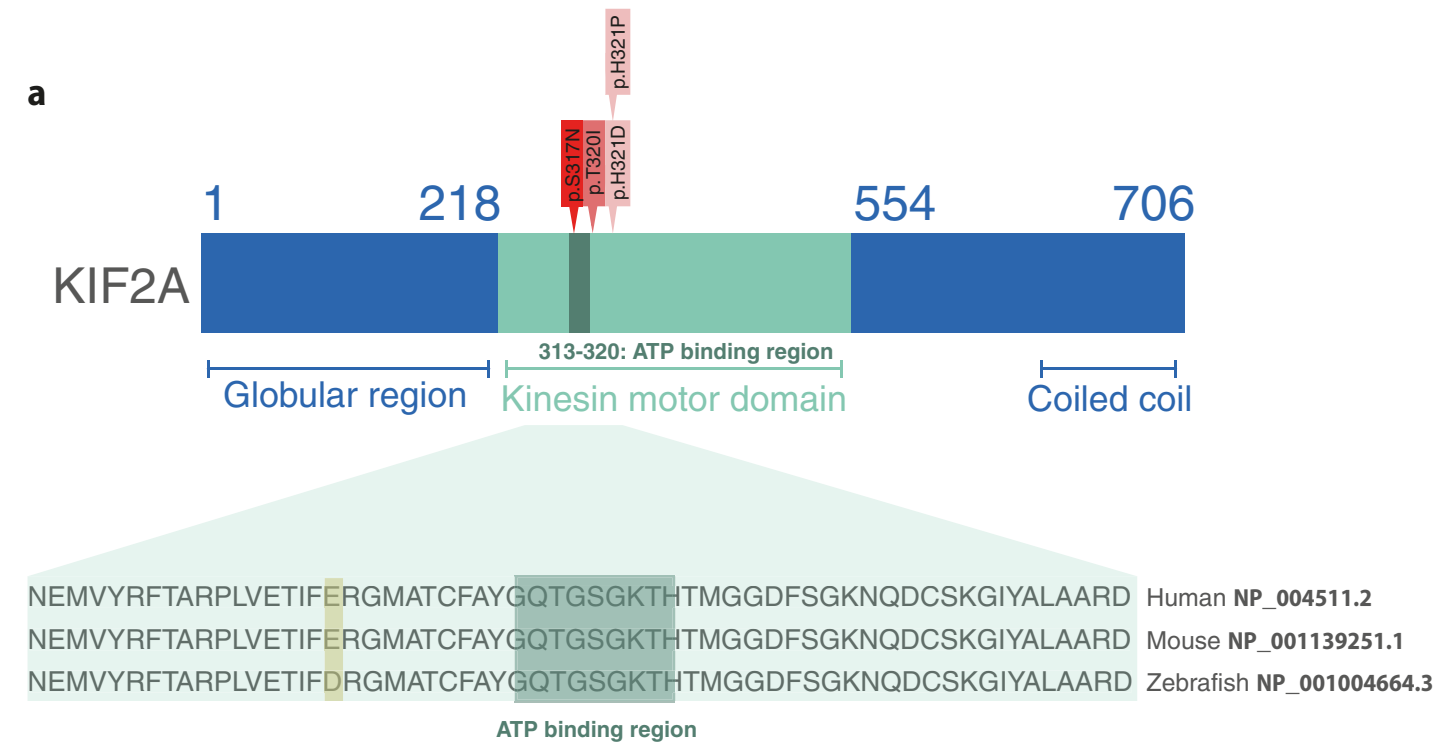

b

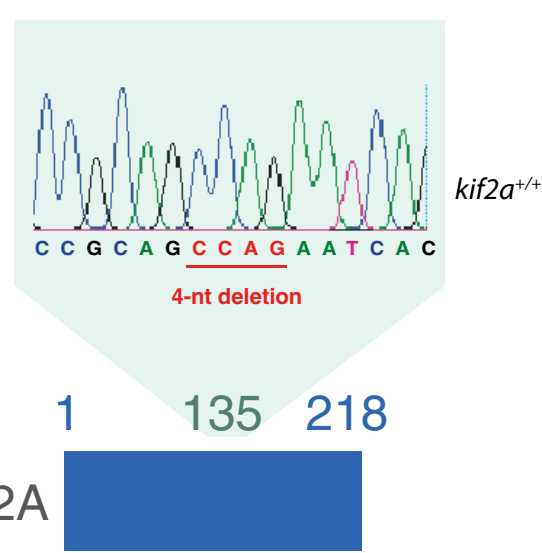

Globular region c

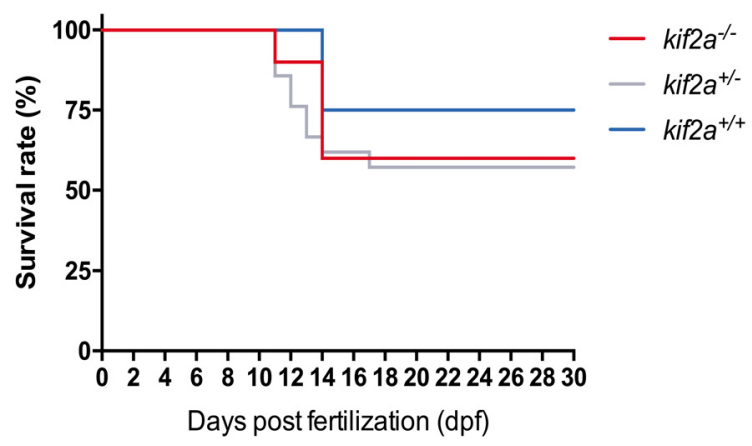

d
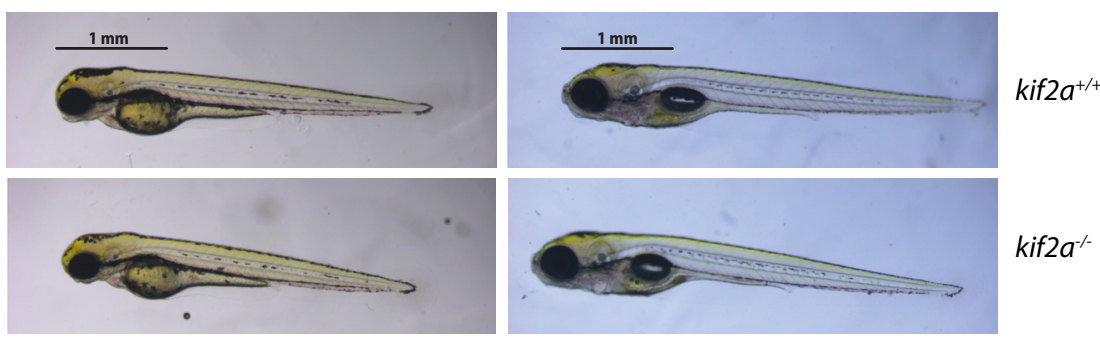

$3 \mathrm{dpf}$

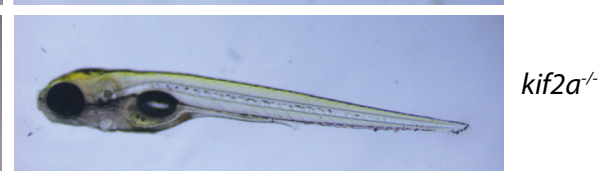

$5 \mathrm{dpf}$

Figure 1. A novel kif2a knock-out zebrafish model shows that kif2a is not indispensable for survival and mutations do not result in gross morphologic abnormalities. a, Linear representation of the human kinesin 2A polypeptide with highlighted four de novo MCDassociated mutations (indicated in red-pink) located in or near the ATP-binding region (indicated in green). Kinesin motor domain, coiled coil, and globular domains (UniProtKB-O00139) are highlighted. Multiple sequence alignment shows the level of conservation of the ATP binding region (indicated in dark turquoise) of human, mouse, and zebrafish. $\boldsymbol{b}$, Localization of 4-nucleotide deletion (CCAG) in the globular region of Kif2a and electropherogram of kif2a ${ }^{+/+}$larvae, confirming the presence of the mutation in homozygotes. $\boldsymbol{c}$, Kaplan-Meier survival rate curves of kif2a ${ }^{+/+}(n=5)$, kif2a $^{+/-}(n=21)$, and kif2a ${ }^{-1-}(n=10)$ larvae. Larvae were obtained by the mating of heterozygous adults. Surviving larvae were counted every $24 \mathrm{~h}$ until $30 \mathrm{dpf}$. $\boldsymbol{d}$, Lateral bright-field images representing 
continued

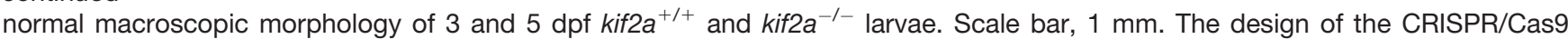
kif2a zebrafish knock-out line is illustrated in Extended Data Figure 1-1. qPCR analysis of kif2c expression levels showed no upregulation of $k i f 2 c$ during development in kif2a $^{-1-}$ larvae (Extended Data Fig. 1-1).

$0.06,029, n=3 ; p<0.0001$, ANOVA, Dunnett's multiplecomparisons test; Fig. 2a,b).

To understand the potential function of kif2a during development, its spatial expression patterns in $\mathrm{kif}_{2} \mathrm{a}^{+/+}$and $k_{i f 2 a^{-1-}}$ larvae were examined. The $8 \mathrm{~h}$ postfertilization (hpf) kif2a mRNA was found to be equally expressed in the whole embryo. At $23 \mathrm{hpf}$, the expression was limited to the head, and gradually became restricted to midbrain and hindbrain at $2 \mathrm{dpf}$. At $5 \mathrm{dpf}$, kif2a mRNA was observed only in the hindbrain (Fig. 2c). In kif2a ${ }^{-1-}$ larvae, no mRNA expression was detected at any developmental stage examined (Fig. 2c). This suggests nonsense-mediated mRNA decay (NMD) because of the mutation resulting in a premature termination codon and thus the total absence of mRNA. Additionally, we also confirmed that there was an absence of protein in kif2a ${ }^{-/}$larvae at 5 and $7 \mathrm{dpf}$, confirming the efficiency of the knock-out (Fig. 2d).

\section{kif2a $^{-/-}$larvae display neurobehavioral alterations like hypoactivity, habituation learning deficits, and increased locomotor behavior to PTZ}

To investigate the impact of Kif2a loss of function on behavioral activity, locomotor experiments were performed on 5-8 dpf kif2a $\mathrm{a}^{+/+}$and kif2a $\mathrm{a}^{-/-}$larvae subjected to a light/dark stimulus. kif2a $a^{-1-}$ larvae showed significantly decreased swimming activity during both the light and dark phases from 6-8 dpf (6 dpf: light, 6389.083 \pm 4322.520, $n=48, p<0.0001$; dark, 29,666.960 $\pm 10,566.630$, $n=48, p<0.0001 ; 7$ dpf: light, 5642.745 $\pm 4051.766, n=48$, $p=0.0194$; dark, 20,825.320 $\pm 11,267.100, n=48, p=0.0019$; 8 dpf: light, 3665.851 $\pm 2489.406, n=47, p=0.0452$; dark, $13,710.230 \pm 6626.408, n=47, p=0.0004$, Student's $t$ test; Fig. 3a) compared with their kif2a ${ }^{+/+}$siblings (6 dpf: light, $11,400.170 \pm 7202.550, \quad n=48 ;$ dark, 40,244.920 \pm 13,768.350, $n=48 ; 7$ dpf: light, $7773.229 \pm 4651.655$, $n=48$; dark, 28,379.650 $\pm 11,812.280, n=48$; $8 \mathrm{dpf}$ : light, $4793.500 \pm 2904.615, n=48$; dark, 20,260.730 \pm $10,363.520, n=48)$. At $5 \mathrm{dpf}$, however, no significant decrease was observed between the kif2a $\mathrm{a}^{-j-}$ larvae (light: 6705.521 $\pm 5427.908, n=48, p=0.9174$; dark: $37,165.790 \pm 14,433.510, n=48, p=0.5394$, Student's $t$ test; Fig. 3a) and kif2a ${ }^{+/+}$larvae (light: 6578.854 \pm 6460.863, $n=48$; dark: 39,130.670 $\pm 16,734.680, n=48$ ).

Moreover, since we observed hypoactivity in kif2a ${ }^{-1-}$ larvae, we investigated whether they presented increased susceptibility to seizures by the administration of a chemical convulsant. kif2a ${ }^{-/-}$animals were incubated with a subthreshold dose of PTZ, a GABA $A$ receptor antagonist that is widely used to induce acute seizures in zebrafish (Afrikanova et al., 2013). We found a significant increase in epileptiform activity in kif2a ${ }^{-1-}$ larvae $(33,431 \pm 12,315, n=20, p=0.0004$, Student's $t$ test; Fig. $3 b$ ) after PTZ incubation in comparison to their $\mathrm{kif2a}^{+/+}$siblings $(19,807 \pm 12,194, n=20)$, confirming increased seizure susceptibility.

Additionally, to evaluate whether kif2a ${ }^{-1-}$ larvae deriving from heterozygous parents had a similar phenotype, we performed the same experiments on heterozygous

Table 1: Statistical table

\begin{tabular}{|c|c|c|c|}
\hline Figure & Data structure & Type of test & Power \\
\hline $1 c$ & NA & Log-rank Mantel-Cox & 0.05 (95\% confidence interval) \\
\hline $1-1 c$ & Gaussian distribution & Unpaired Student's $t$ test & 0.05 (95\% confidence interval) \\
\hline $1-1 d$ & Gaussian distribution & Unpaired Student's $t$ test & 0.05 (95\% confidence interval) \\
\hline $2 a$ & Gaussian distribution & One-way ANOVA with Dunnett's multiple-comparisons test & 0.05 (95\% confidence interval) \\
\hline $2 b$ & Gaussian distribution & One-way ANOVA with Dunnett's multiple-comparisons test & 0.05 (95\% confidence interval) \\
\hline $3 a$ & Gaussian distribution & Unpaired Student's $t$ test per day & 0.05 (95\% confidence interval) \\
\hline $3 b$ & Gaussian distribution & Unpaired Student's $t$ test & 0.05 (95\% confidence interval) \\
\hline $3 c$ & Gaussian distribution & Unpaired Student's $t$ test & 0.05 (95\% confidence interval) \\
\hline $3 d$ & Gaussian distribution & Unpaired Student's $t$ test & 0.05 (95\% confidence interval) \\
\hline $3-1 a$ & Gaussian distribution & One-way ANOVA with Dunnett's multiple-comparisons test per day & 0.05 (95\% confidence interval) \\
\hline $3-1 b$ & Gaussian distribution & One-way ANOVA with Dunnett's multiple-comparisons test & 0.05 (95\% confidence interval) \\
\hline $3-1 c$ & Gaussian distribution & One-way ANOVA with Dunnett's multiple-comparisons test & 0.05 (95\% confidence interval) \\
\hline $4 b$ & Gaussian distribution & Unpaired Student's $t$ test & 0.05 (95\% confidence interval) \\
\hline $4 c$ & Gaussian distribution & Unpaired Student's $t$ test & 0.05 (95\% confidence interval) \\
\hline $4-1 a$ & Gaussian distribution & One-way ANOVA with Dunnett's multiple-comparisons test & 0.05 (95\% confidence interval) \\
\hline $4-1 b$ & Gaussian distribution & One-way ANOVA with Dunnett's multiple-comparisons test & 0.05 (95\% confidence interval) \\
\hline $5 a-d$ & Gaussian distribution & Unpaired Student's $t$ test & 0.05 (95\% confidence interval) \\
\hline $5 f$ & Gaussian distribution & Unpaired Student's $t$ test & 0.05 (95\% confidence interval) \\
\hline $5 g, h$ & Gaussian distribution & Unpaired Student's $t$ test & 0.05 (95\% confidence interval) \\
\hline $5-1 b$ & Gaussian distribution & Unpaired Student's $t$ test & 0.05 (95\% confidence interval) \\
\hline $5-1 c-h$ & Gaussian distribution & Unpaired Student's $t$ test & 0.05 (95\% confidence interval) \\
\hline $6 b$ & Gaussian distribution & Unpaired Student's $t$ test & 0.05 (95\% confidence interval) \\
\hline $6 e$ & Gaussian distribution & Unpaired Student's $t$ test & 0.05 (95\% confidence interval) \\
\hline $6 h$ & Gaussian distribution & Unpaired Student's $t$ test & 0.05 (95\% confidence interval) \\
\hline
\end{tabular}



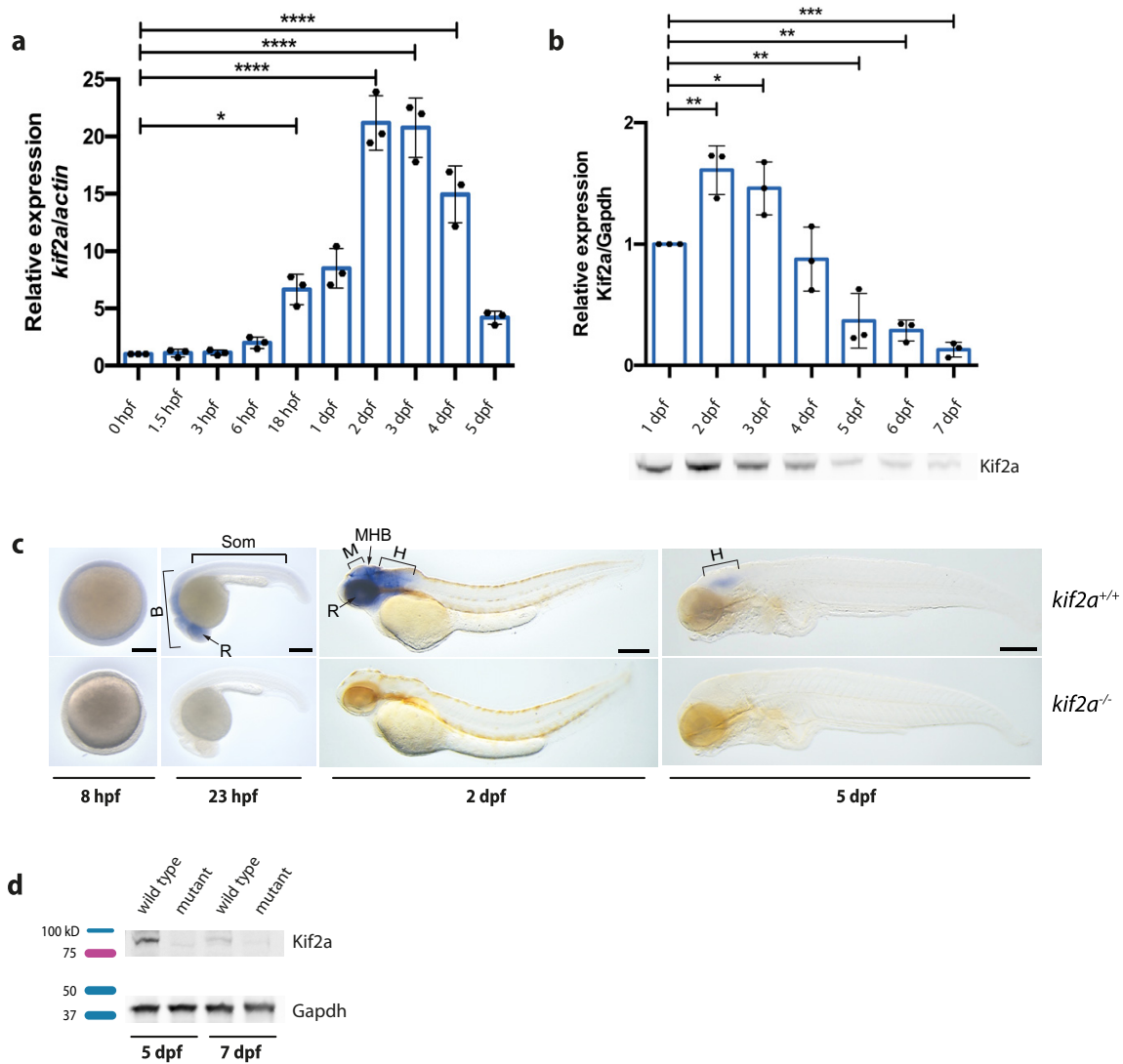

Figure 2. Expression and localization pattern of kif2a in the developing zebrafish. a, qPCR analysis of kif2a levels in kif2a ${ }^{+/+}$larvae normalized to actin and represented as the fold change expression to $0 \mathrm{hpf}$. Values are reported as the mean \pm SEM of three separate experiments. Significant values are noted as ${ }^{* * *} p \leq 0.0001$ and ${ }^{*} p \leq 0.05$. $\boldsymbol{b}$, Relative quantification of Kif2a protein expression

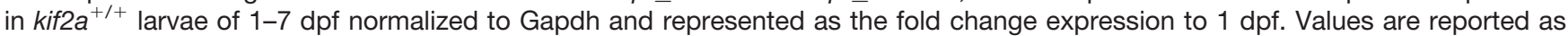
the mean \pm SEM of three separate experiments. Significant values are noted as ${ }^{\star * *} p \leq 0.001,{ }^{* *} p \leq 0.01$, and ${ }^{*} p \leq 0.05$. Below the graph is a representative Western blot image of Kif2a protein expression levels in kif2a ${ }^{+/+}$larvae of $1-7$ dpf. $\boldsymbol{c}$, Spatiotemporal expression patterns of kif2a by whole-mount RNA in situ hybridization at 8 hpf, 23 hpf, 2 dpf, and 5 dpf. B, Brain; H, hindbrain; M, midbrain; $\mathrm{MBH}$, midbrain-hindbrain boundary; Som, somite; R, retina. Scale bar, $200 \mu \mathrm{m}$. $\boldsymbol{d}$, Representative Western blot image of Kif2a protein expression levels comparing kif2a ${ }^{+/+}$with $\mathrm{kif}^{2} \mathrm{a}^{-/-}$larvae at 5 and $7 \mathrm{dpf}$.

offspring. Our results demonstrated that there was no difference at any day postfertilization between the swimming

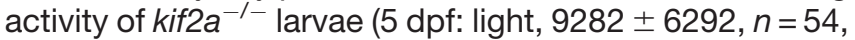
$p=0.6908 ; \quad$ dark, $48,504 \pm 14,603, \quad n=54, \quad p=0.7322$; 6 dpf: light, $8405 \pm 5250, n=55, p=0.0671$; dark, 39,557 \pm 13,571, $n=55, p=0.5420 ; 7$ dpf: light, $6258 \pm 4875, n=55$, $p=0.3670$; dark, 29,524 $\pm 12,700, n=55, p=0.8121 ; 8 \mathrm{dpf}$ : light, $4707 \pm 3545, \quad n=55, \quad p=0.7292 ;$ dark, $19,812 \pm$ $11,920, n=55, p=0.0 .2895$, ANOVA, Dunnett's multiplecomparisons test; Extended Data Fig. 3-1a) compared with their kif2a ${ }^{+/+}$siblings (5 dpf: light, $8208 \pm 7027, n=51$; dark, $46,291 \pm 16,613, n=51 ; 6$ dpf: light, $6242 \pm 4024, n=51$; dark, $36,549 \pm 16,230, n=51 ; 7$ dpf: light, $5757 \pm 4283$, $n=51$; dark, 28,702 $\pm 13,452, n=51$; 8 dpf: light, $4329 \pm$ $3365, n=51$; dark, $16,375 \pm 11,071, n=51$ ). Similarly, $\mathrm{kif2a}^{+/-}$larvae did not do not present with an abnormal behavioral phenotype ( 5 dpf: light, $8418 \pm 7369, n=117$; dark, 48,354 $\pm 17,752, n=117$; 6 dpf: light, $7370 \pm 4807, n=117$; dark, $38,840 \pm 14,707, n=117 ; 7$ dpf: light, $6800 \pm 4390$, $n=116$; dark, 30,264 $\pm 15,938, n=116$; 8 dpf: light, $4792 \pm$ 3529, $n=117$; dark, $19,030 \pm 12,283, n=117$ ). Overall, these results confirm that an offspring from homozygous parents is needed to have the full-blown phenotype.

Since MCDs are an important cause of developmental delay and cognitive deficits (Desikan and Barkovich, 2016; Represa, 2019), we also examined whether kif2a ${ }^{-/-}$ larvae had difficulties in acquiring, storing, and recalling learned information by performing a habituation learning assay (Wolman et al., 2011). During the experiment, larvae were subjected to a spaced training consisting of multiple dark flashes (DFs) alternated with $10 \mathrm{~min}$ light periods and, after a pause, were further exposed to a test assay consisting of a block of DFs (Fig. 3c). kif2a ${ }^{+/+}$ $(47.82 \pm 31.70, n=87)$ and $\mathrm{kif}^{-1-} \mathrm{a}^{-1}(51.41 \pm 21.77, n=88$, $p=0.3833$, Student's $t$ test; Fig. $3 d$ ) larvae showed an adaptation profile similar to DFs during the habituation, as quantified by calculation of the habituation percentage. Strikingly, $\mathrm{kif2a}^{-/}$larvae $(1.723 \pm 0.7958, \quad n=30$, $p=0.0053$, Student's $t$ test; Fig. $3 e$ ) demonstrated memory deficits as they were not able to respond to DFs in the same way as their $\mathrm{kif2a}^{+/+}$siblings $(1.212 \pm 0.4738$, $n=27$ ) during the training, after a period of inactivation. 


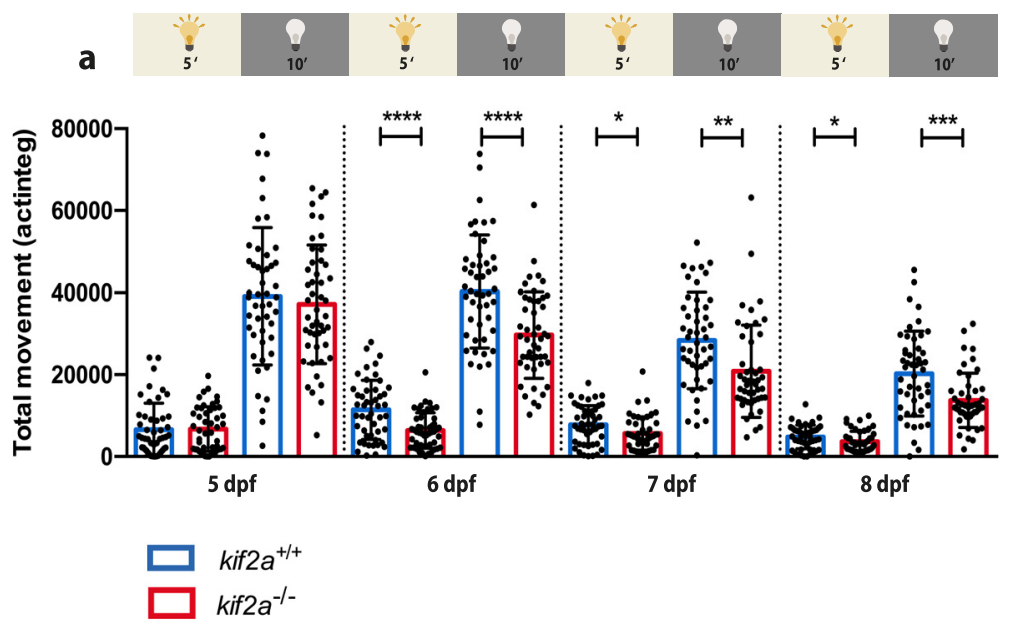

b

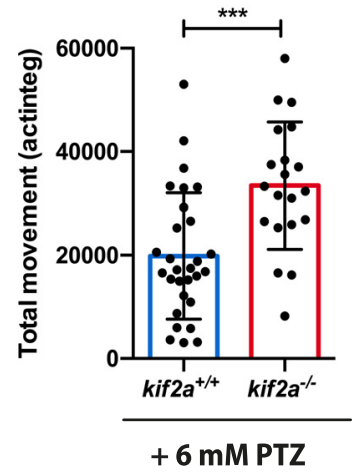

C

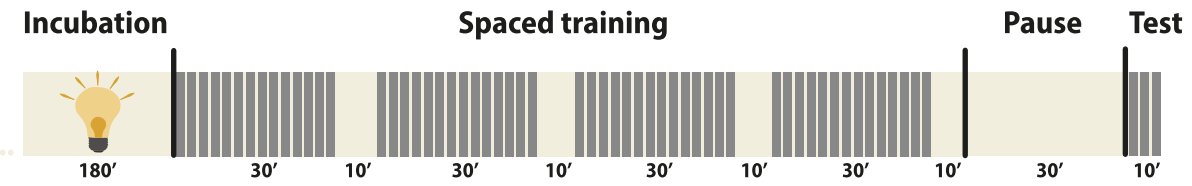

d
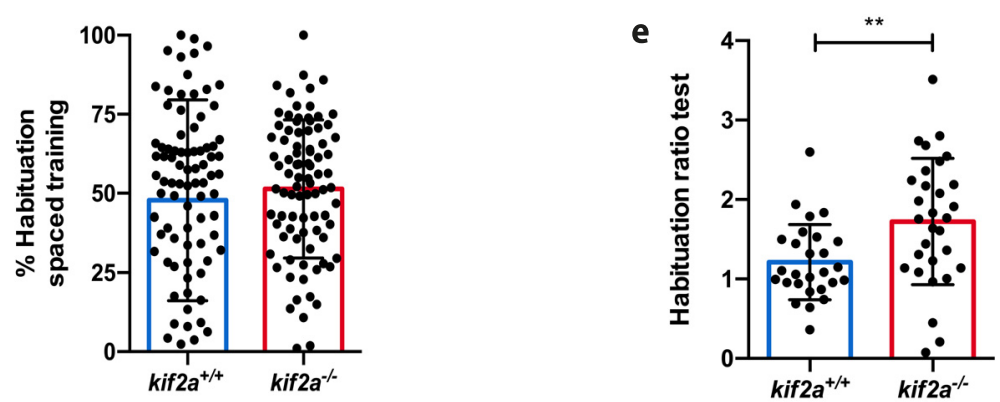

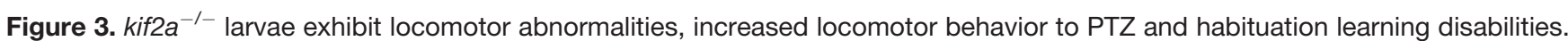
a, Average total movement of $k i f 2 a^{+/+}$and $k i f 2 a^{-/-}$larvae from 5 to $8 \mathrm{dpf}$ expressed in actinteg units. Values are reported as the mean \pm SD of four separate experiments. Significant values are noted as ${ }^{* * *} p \leq 0.0001,{ }^{* *} p \leq 0.001,{ }^{* *} p \leq 0.01$, and ${ }^{*} p \leq 0.05$. $\boldsymbol{b}$, Average total movement of $5 \mathrm{dpf} \mathrm{kif}_{2} \mathrm{a}^{+/+}$and kif $2 \mathrm{a}^{-/-}$larvae after $6 \mathrm{~mm}$ PTZ immersion for $30 \mathrm{~min}$. Values are reported as the mean $\pm \mathrm{SD}$ of four separate experiments. Significant values are noted as ${ }^{* \star *} p \leq 0.001$. $c$, The habituation learning assay performed on 6

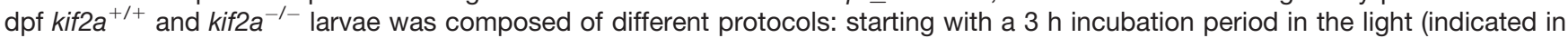
yellow), followed by a habituation training consisting of four periods with 120 DFs with a $15 \mathrm{~s}$ ISI (regions with gray and yellow stripes), alternated by $10 \mathrm{~min}$ of light, a period of $30 \mathrm{~min}$ pause in the light preceding the actual test, and a test consisting of 10 DFs with a $60 \mathrm{~s}$ ISI (region with gray and yellow stripes). $\boldsymbol{d}$, Percentage habituation of kif2a $\mathrm{a}^{+/+}$and kif2a $\mathrm{a}^{-1-}$ larvae to DFs during the spaced training. Values are reported as the mean \pm SD of three separate experiments. e, Habituation ratio of average movement of kif2a $^{+/+}$and kif2a $^{-1-}$ larvae to DFs during the test. Values are reported as the mean \pm SD of three separate experiments. Significant values are noted as ${ }^{* *} p \leq 0.01$. No locomotor abnormalities and habituation learning disabilities were observed in kif2a ${ }^{-1-}$ zebrafish larvae from +/- incrosses (Extended Data Fig. 3-1).

These results demonstrate that kif2a deficiency results in disability in habituation, suggesting memory decay and learning disabilities.

Correspondingly, to characterize the phenotype of $\mathrm{kif2a}^{-1-}$ larvae deriving from heterozygous parents, this experiment was performed on heterozygous offspring (Extended Data Fig. 3-1b-d). Both kif2a ${ }^{+/-}$(percentage of habituation: $42.39 \pm 35.83 \%, n=66$; habituation ratio: $1.161 \pm 0.6025, n=30$ ) and $\mathrm{kif2a}^{-1-}$ larvae (percentage of habituation: $35.92 \pm 33.74 \%, n=39$; $p=0.5279$, ANOVA, Dunnett's multiple-comparisons test; Extended Data Fig. 3-1c; habituation ratio: $1.216 \pm 0.5361, n=30, p=0.9423$, ANOVA, Dunnett's multiple-comparisons test; Extended Data Fig. 3-1d) showed a habituation and learning profile similar to that of their $\mathrm{kif}_{2} \mathrm{a}^{+/+}$siblings (percentage of habituation: $35.76 \pm 39.16, \quad n=22$; habituation ratio: $1.205 \pm 0.7903, n=29$ ), confirming that an offspring 
a
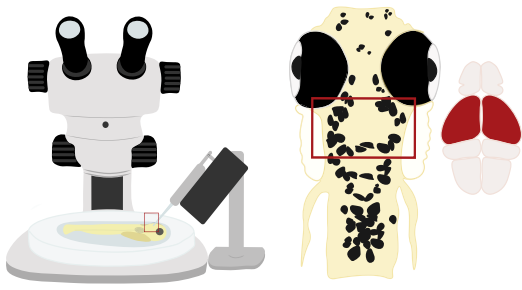

C

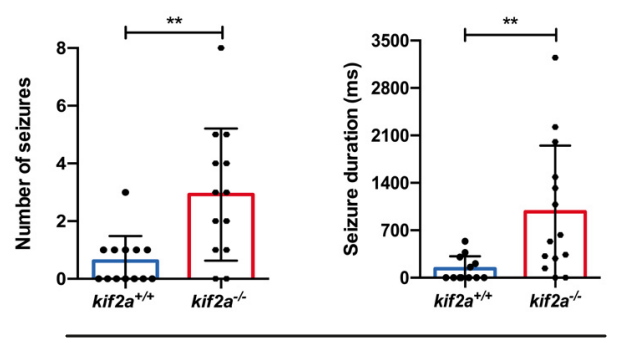

$+6 \mathrm{mM}$ PTZ b
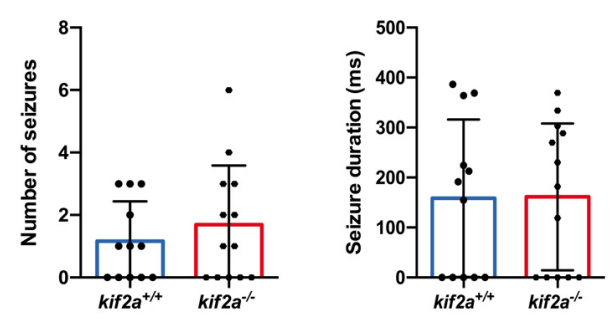

d

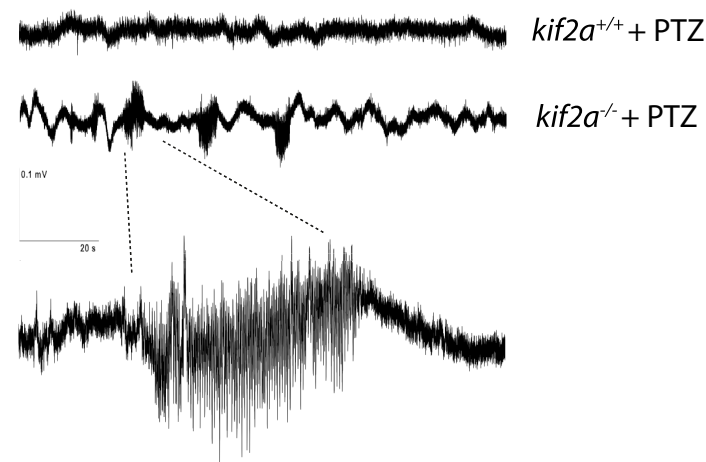

0.02

Figure 4. $\mathrm{kif}_{2} \mathrm{a}^{-/-}$larvae show increased susceptibility to epilepsy. a, Visual representation of the setup of local field potential recording from optic tectum (indicated in red) of $5 \mathrm{dpf}$ larvae. $\boldsymbol{b}$, Number of seizures and average seizure duration in kif2a ${ }^{+/+}$and $k_{i f 2 a^{-l-}}$ larvae. $\boldsymbol{c}$, Number of seizures and average seizure duration in kif2a ${ }^{+/+}$and $k i f 2 a^{-1-}$ larvae after 6 mM PTZ immersion for

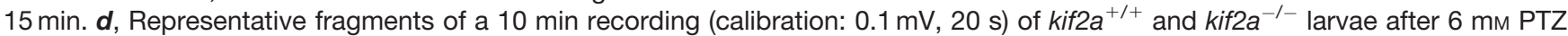
immersion for $15 \mathrm{~min}$, with enlargement of a polyspiking event (calibration: $0.02 \mathrm{mV}, 2 \mathrm{~s}$ ) in kif2a ${ }^{-/-}$larvae. Data are represented as the mean \pm SD. Significant values are noted as ${ }^{* *} p \leq 0.01$. No increased seizure susceptibility was observed in the brains of $k i f 2 a^{-1}$ zebrafish larvae from +/- incrosses (Extended Data Fig. 4-1).

from homozygous adults is needed to obtain a fullblown phenotype.

\section{$\mathrm{kif2a}^{-/-}$larvae present increased seizure susceptibility to PTZ}

To investigate whether kif2a knockout resulted in abnormal epileptic brain activity, noninvasive local field potential recordings were performed from the optic tecta of $5 \mathrm{dpf} \mathrm{kif2a}^{+/+}$and $\mathrm{kif2a}^{-/-}$larvae (Fig. 4a). Events were classified as epileptiform if the amplitude equaled or exceeded three times the baseline. No significant increase in abnormal spontaneous epileptiform activity was detected in kif2a-l- larvae (seizure number: $1.692 \pm 1.888$, $n=13, p=0.4262$, Student's $t$ test; Fig. $4 b$, left; seizure duration: $430.0 \mathrm{~ms} \pm 494.3, n=13, p=0.0692$, Student's $t$ test; Fig. $4 b$, right) compared with $k_{i f 2 a^{+/+}}$larvae (seizure number: $1.167 \pm 1.267, n=12$; seizure duration: $104.2 \mathrm{~ms} \pm 127.6, n=9)$. We then tried to determine whether $\mathrm{kif}^{2} \mathrm{a}^{-\prime-}$ larvae were presenting increased susceptibility to seizures by the administration of a convulsant. $\mathrm{kif}^{2} \mathrm{a}^{-/-}$animals were incubated with a subthreshold dose of PTZ. We found a significant increase in epileptiform activity in kif2a ${ }^{-1-}$ larvae after PTZ incubation (seizure number: $2.923 \pm 2.29, n=13, p=0.0024$, Student's $t$ test; Fig. 4c, left; seizure duration: $2360 \mathrm{~ms} \pm 2723, n=12$, $p=0.0120$, Student's $t$ test; Fig. $4 c$, right) compared with their $\mathrm{kif2a}^{+/+}$siblings (seizure number: $0.6154 \pm 0.8697$, $n=13$; seizure duration: $94.59 \mathrm{~ms} \pm 141.5, n=11)$, confirming increased seizure susceptibility (Fig. 4c, d).

Again, we evaluated whether kif2a $a^{-1-}$ larvae deriving from heterozygous parents presented with spontaneous abnormal epileptic brain activity or increased PTZ seizure susceptibility. Neither $\mathrm{kif2a}^{+/-}$(seizure number: $0.5 \pm 0.6325, n=16, p=0.1953$; seizure duration: 114.0 $\mathrm{ms} \pm 148.7, n=17)$ and kif2a $^{-1-}$ larvae (seizure number: $0.0 \mathrm{~ms} \pm 0.0, n=7, p=0.1953$, ANOVA, Dunnett's multiple-comparisons test; Extended Data Fig. 4-1a, left; seizure duration: $0.0 \pm 0.0, n=7, p=0.0759$, ANOVA, Dunnett's multiple-comparisons test; Extended Data Fig. 4-1a, right) showed any spontaneous abnormal epileptiform brain activity compared with their $\mathrm{kif2a}^{+/+}$siblings (seizure number: $0.3750 \pm 0.744, n=8$; seizure duration: $36.44 \mathrm{~ms} \pm 73.76, n=8$ ). Similarly, no significantly increased PTZ seizure susceptibility was observed in kif2a $^{+/-}$larvae (seizure number: $1.545 \pm 1.968, n=11$, $p=0.3183$; seizure duration: $63.61 \mathrm{~ms} \pm 80.90, n=9$ ) and kif2a $^{-1-}$ larvae (seizure number: $1.4 \pm 2.271, n=10, p=$ 0.3183 , ANOVA, Dunnett's multiple-comparisons test; Extended Data Fig. 4-1b, left; seizure duration: $160.9 \mathrm{~ms}$ $\pm 304, n=9, p=0.3332$, ANOVA, Dunnett's multiple-comparisons test; Extended Data Fig. 4-1b, right) compared 
a

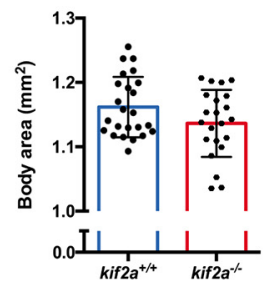

C

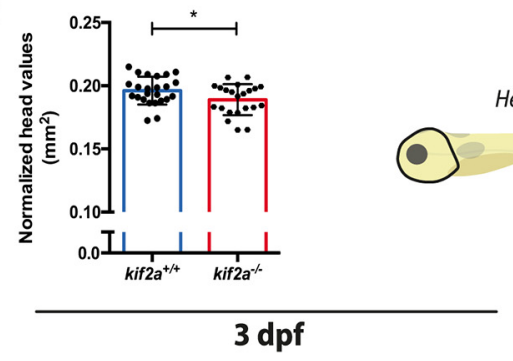

e
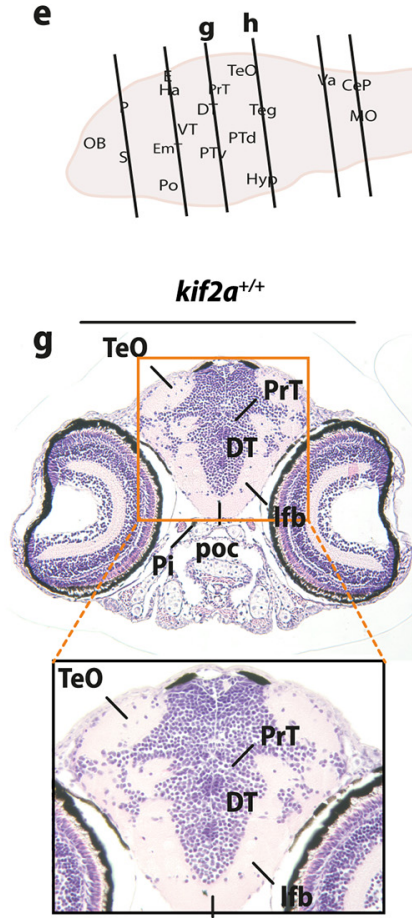

poc

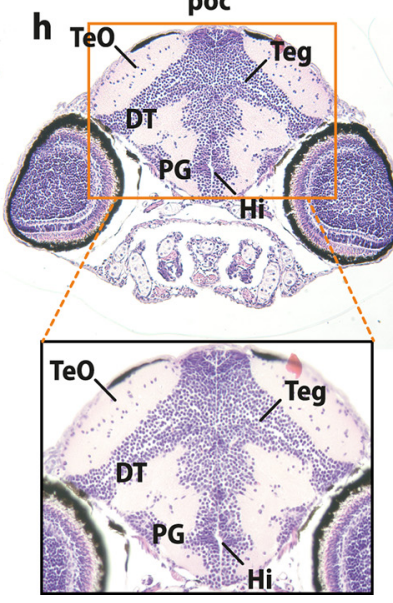

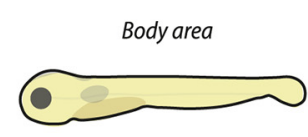

Head area
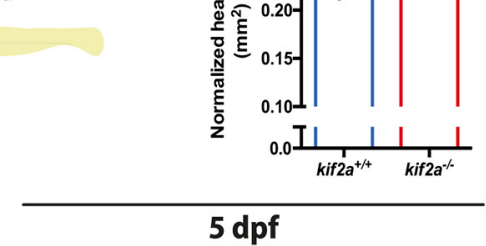

f
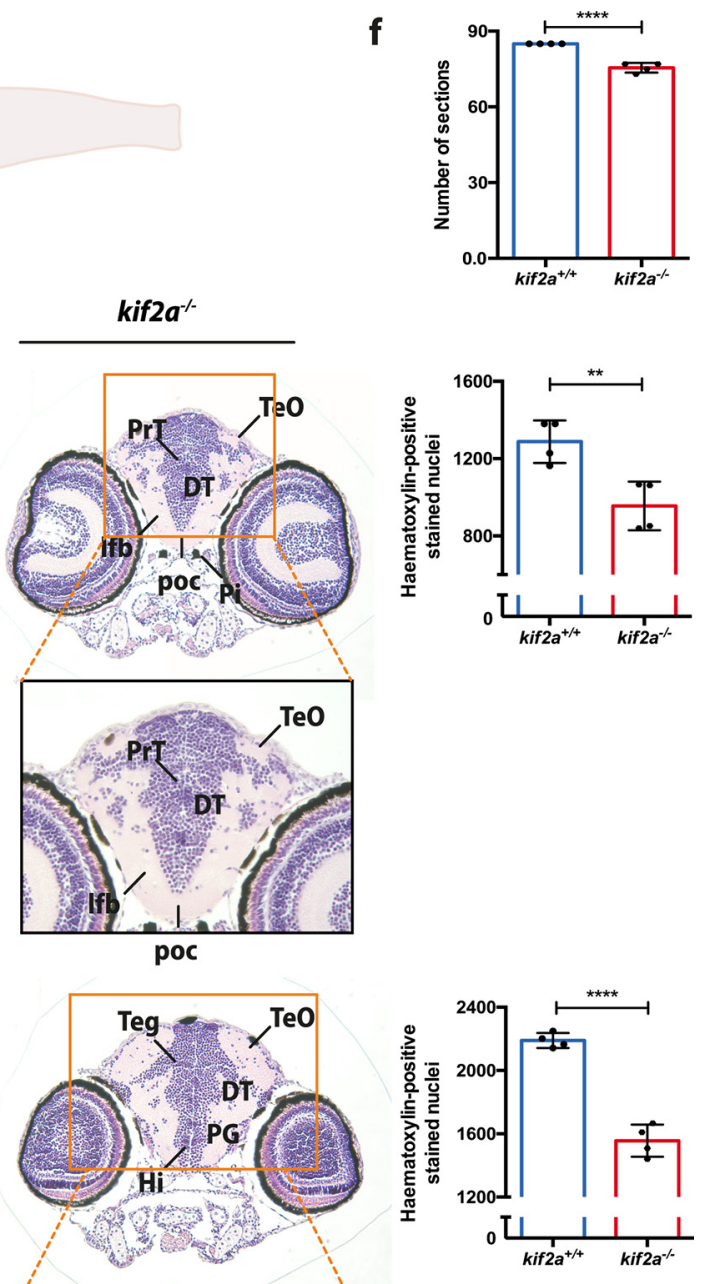
continued

Figure 5. kif2a larvae present microcephaly that is associated with defects in neurologic development. a-d, Comparison of the individual measurements for body area $(\boldsymbol{a}, \boldsymbol{b})$ and head size $(\boldsymbol{c}, \boldsymbol{d})$ for kif $2 \mathrm{a}^{+/+}$and $\mathrm{kif2}^{-1-}$ larvae at 3 and 5 dpf. Data are represented as the mean $\pm \mathrm{SD}$. Significant values are noted as ${ }^{*} p \leq 0.05$. e, Histologic assessment of 5 dpf kif2a ${ }^{-/-}$and kif2a ${ }^{+/+}$larval brains. Six brain regions (from forebrain to hindbrain) were selected per genotype as indicated in the diagram. $\boldsymbol{f}$, Comparison of the number of brain sections from forebrain to hindbrain (e) between $k i f 2 a^{+/+}$and $k i f 2 a^{-1-}$ larvae. Data are represented as the mean \pm SD. Significant values are noted as ${ }^{* \star *} p \leq 0.0001 . \boldsymbol{g}, \boldsymbol{h}$, Coronal sections stained with H\&E imaged at $20 \times$ and $40 \times$ (indicated by orange square) magnification. DT, Dorsal thalamus; Hi, intermediate hypothalamus; Ifb, lateral forebrain bundle; PG, preglomerular complex; PrT, pretectum; Teg, midbrain tegmentum; TeO, tectum opticum. Bar graphs compare hematoxylin-positive stained nuclei between $k_{i f 2 a^{+/+}}$and kif2a $a^{-1-}$ larvae. Data are represented as the mean \pm SD. Significant values are noted as ${ }^{* \star *} p \leq 0.0001$ and ${ }^{* *} p \leq 0.01$.

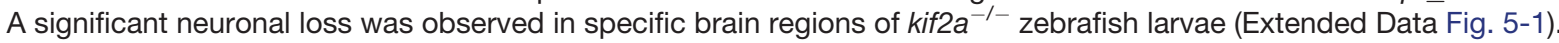

with their kif2a ${ }^{+/+}$siblings (seizure number: $0.0 \mathrm{~ms} \pm 0.0$, $n=5$; seizure duration: $0.0 \mathrm{~ms} \pm 0.0, n=5$ ).

\section{Aberrant head morphology of kif2a mutants is associated with defects in neurologic development}

To investigate whether the loss of function of kif2a was associated with microcephaly, one of the clinical symptoms of patients with KIF2A mutations (Tian et al., 2016; Cavallin et al., 2017), head surface areas of 3 and $5 \mathrm{dpf}$ $\mathrm{kif}_{2} \mathrm{a}^{-1-}$ larvae were measured and normalized against total body surface. At $3 \mathrm{dpf}$, a statistically significant change in head size was observed between kif2a ${ }^{+/+}$ $(0.1961 \pm 0.01106, n=24)$ and $k_{i f 2 a}{ }^{-/}$zebrafish larvae $(0.1890 \pm 0.01226, n=22 ; p=0.0427$, Student's $t$ test; Fig. 5c) that was also present in $5 \mathrm{dpf} \mathrm{kif2a^{+/+ }}(0.2321 \pm$ $0.01094, n=19)$ and kif2a $a^{-1-}$ larvae $(0.2252 \pm 0.006116$, $n=17 ; p=0.0295$, Student's $t$ test; Fig. $5 d$ ). Body area did not differ significantly between the larval groups at both 3 dpf $\left(\mathrm{kif2a}^{+/+}: 1.162 \pm 0.047, n=24 ; \mathrm{kif2a}^{-/-}: 1.136 \pm 0.052\right.$, $n=22 ; p=0.0899$, Student's $t$ test; Fig. $5 a)$ and $5 \mathrm{dpf}\left(\mathrm{kif2a}^{+/+}\right.$: $1.321 \pm 0.066, n=19 ; k_{i f 2 a}{ }^{-/-}: 1.358 \pm 0.056, n=17 ; p=$ 0.0801 , Student's $t$ test; Fig. $5 b$ ).

Moreover, enlarged ventricles and cerebellar anomalies have been described as common features in Kif2a-null mice (Homma et al., 2003). To investigate whether microcephaly was caused by dysmorphologies in the brain and whether enlarged ventricles and cerebellar abnormalities were among the characteristics of our model, histologic examination of $5 \mathrm{dpf}$ zebrafish brain tissue sections was performed (Fig. 5e, Extended Data Fig. 5-1a-h). Although zebrafish kif2a ${ }^{-1-}$ larvae did not present enlarged ventricles relative to the brain tissue, we found significantly fewer brain sections for kif2a $^{-1-}$ larvae $(75.5 \pm 1.915$, $n=4 ; p<0.0001$, Student's $t$ test) from forebrain to hindbrain (Fig. 5e,f, diagram) compared with their kif2a $^{+/+}$siblings $(85.0 \pm 0.0, n=4)$.

In addition, we observed that there were significantly fewer hematoxylin-positive stained nuclei detected in specific brain regions (Fig. 5e, $g$ and $h$ ) of kif2a $^{-/-}$larvae (Fig. $5 g, 955.3 \pm 125.7, n=4 ; p=0.0072$, Student's $t$ test; Fig. $5 h, 1556 \pm 101.6, n=4 ; p<0.0001$, Student's $t$ test) than those in their kif2a ${ }^{+/+}$siblings (Fig. 5g, $1288 \pm 109.8$, $n=4$; Fig. $5 h, 2190 \pm 46.99, n=4)$. These findings are in line with a significantly smaller head size for kif2a $\mathrm{a}^{-1-}$ larvae (Fig. $5 c, d$ ). Further examination showed that there were no cerebellar anomalies in the kif2a knock-out zebrafish brain. More particularly, all parts of the cerebellum such as valvula cerebelli and corpus cerebelli (cerebellar plate), together with the granular eminence (a caudolateral third part) were clearly present both in $\mathrm{kif2a}^{+/+}$and $\mathrm{kif2a}^{-/-}$animals (Extended Data Fig. 5-1g,h; Mueller and Wullimann, 2016).

\section{Loss of kif2a is associated with neuronal cell proliferation defects and increased apoptosis}

Having found that a loss of kif2a in zebrafish was associated with decreased head size and neuronal loss, we investigated whether this phenotype could be caused by defects in neuronal cell proliferation or excessive cell death in the brain. Whole-mount BrdU staining in 5 dpf zebrafish larval optic tectum (Fig. 6a) demonstrated that there was a significant decrease of proliferating cells in kif2a $a^{-/-}$brains $(7.684 \pm 3.830, n=19$; $p=0.0145$, Student's $t$ test; Fig. $6 b, c)$ when compared with their $k_{i f 2 a^{+/+}}$siblings $(11.13 \pm 3.907, n=15)$. Caspase-3 immunohistochemistry of $5 \mathrm{dpf}$ larval sections, detecting early-stage apoptosis, showed an increase in the apoptotic cells for kif2a ${ }^{-/-}$larvae $(34.70 \pm 22.31$, $n=24 ; p=0.0033$, Student's $t$ test; Fig. $6 e$ ) from forebrain to hindbrain (Fig. 6d, diagram) compared with their kif2a ${ }^{+/+}$ siblings (16.49 $\pm 16.69, n=22)$. In particular, as kif2a mRNA expression became restricted to hindbrain at $5 \mathrm{dpf}$ (Fig. 2c), we checked whether elevated levels of apoptotic cells could be observed in sections obtained from this region (Fig. 6d, f and $g, f, g$ ). Indeed, we found a significant difference between regions of the hindbrain of $\mathrm{kif2a}^{+/+}(16.61 \pm 18.11$, $n=7)$ and $k_{i f 2 a^{-1-}}$ larvae $(42.71 \pm 17.80, n=8 ; p=0.0147$, Student's $t$ test; Fig. 6h).

\section{Discussion}

Although mutations in tubulin and kinesin genes have been described in various syndromes associated with brain malformations and DRE (Bahi-Buisson and Cavallin, 2016; Poirier et al., 2013; Guerrini and Dobyns, 2014; Tian et al., 2016; Cavallin et al., 2017), and considerable preclinical/clinical knowledge has been obtained (Homma et al., 2003, 2018; Broix et al., 2018), the majority of patients with MCDs and associated DRE remain unresponsive to current pharmacological therapies (Papayannis et al., 2012; Represa, 2019). Therefore, a greater understanding of the mechanisms underlying MCDs is essential to developing more effective therapeutic agents against MCDs. Although mice are common choices for modeling human diseases, zebrafish offer a number of unique advantages of in vitro scalability in a vertebrate model, which is 
a

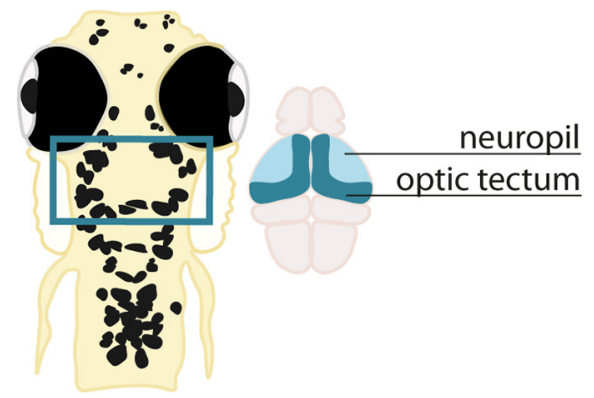

b

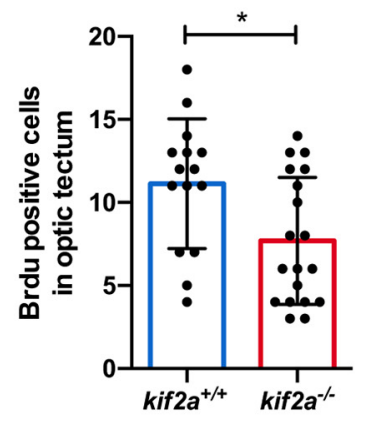

d

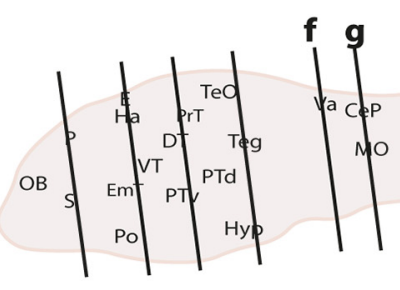

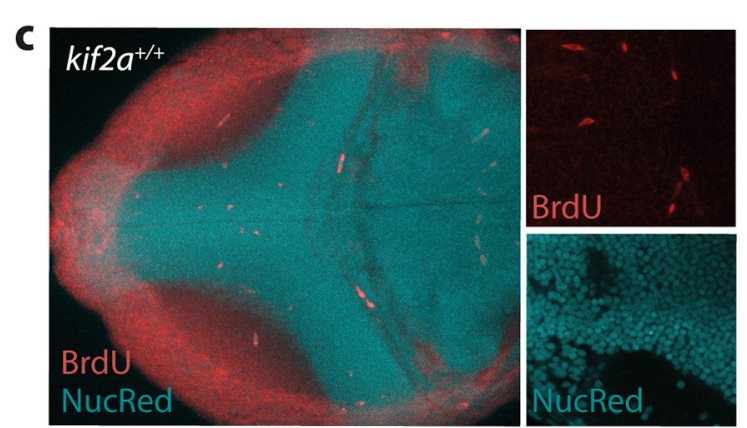

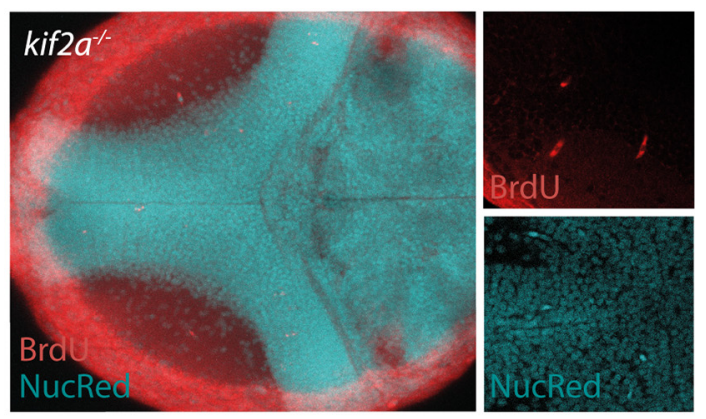

e

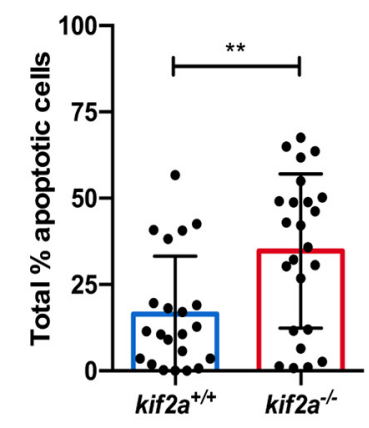

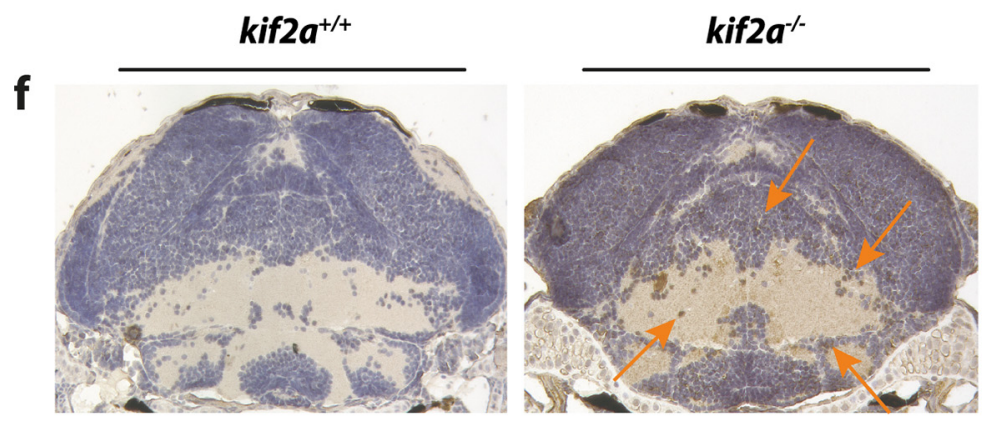

$\mathbf{g}$

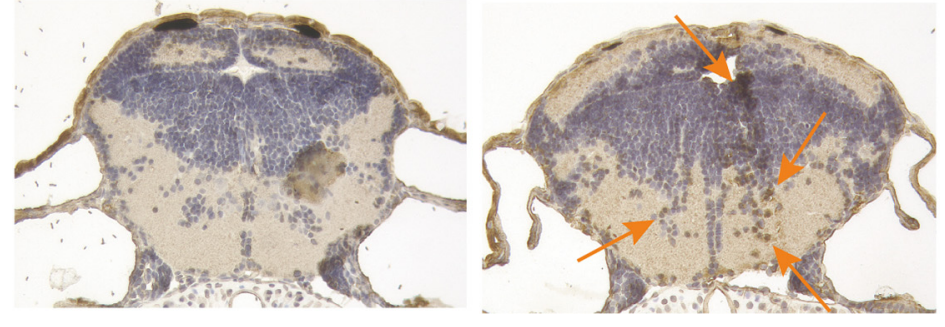

h

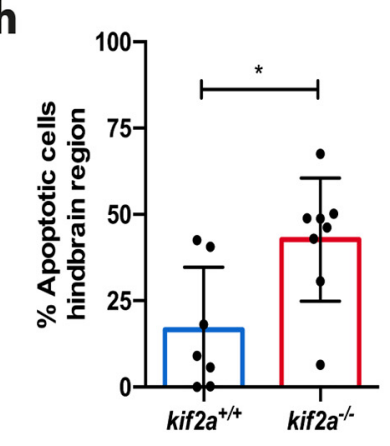


continued

Figure 6. Neuronal cell proliferation defects and increased apoptosis in kif2a $a^{-1-}$ larvae. a, Cell proliferation was measured in the optic tectum of $5 \mathrm{dpf} k i f 2 \mathrm{a}^{+/+}$and $\mathrm{kif}_{2} \mathrm{a}^{-/-}$larvae as indicated in the diagram. $\boldsymbol{b}$, Comparison of the number of proliferating cells in the optic tectum of $\mathrm{kif}_{2} \mathrm{a}^{+/+}$and $\mathrm{kif2a}^{-/-}$larvae showing BrdU-positive cells in dorsal z-stacks. Data are represented as the mean \pm SD. Significant values are noted as ${ }^{*} p \leq 0.1$. c, Representative $z$-stacks of optic tecta with BrdU and nuclei staining of $k i f 2 a^{+/+}$and $k_{i f 2 a^{-/-}}$larvae at $5 \mathrm{dpf}$. d. Histologic assessment of $5 \mathrm{dpf} k i f 2 \mathrm{a}^{-/-}$and $k i f 2 \mathrm{a}^{+/+}$larval brains. Six brain regions (from forebrain to hindbrain) were selected per genotype as indicated in the diagram. e, Total amount of apoptotic cells in the brain of kif2a ${ }^{+/+}$and $k_{i f 2 a} a^{-1}$ larvae. Data are represented as the mean \pm SD. Significant values are noted as ${ }^{* *} p \leq 0.01$. $\boldsymbol{f}, \boldsymbol{g}$, Coronal sections stained against active caspase- 3 , imaged at $20 \times$ magnification. $\boldsymbol{h}$, Percentage of apoptotic cells in the hindbrain region of kif2a ${ }^{+/+}$and $k_{i f 2 a^{-1-}}$ larvae. Data are represented as the mean \pm SD. Significant values are noted as ${ }^{*} p \leq 0.05$.

particularly valued in rapid CNS drug discovery (Stewart et al., 2015; Khan et al., 2017).

The aim of the present study was to advance our understanding of KIF2A-related MCDs and DRE by investigating the pathologic consequences of Kif2a loss of function in a novel zebrafish model. By targeting an early exon of kif2a sequence with CRISPR/Cas9, we generated an outof-frame deletion leading to a premature stop codon, thus blocking downstream translation of Kif2a essential structural domains required for its activity, resulting in a total lack of Kif2a protein in our model. Correspondingly, missense variants described in patients with KIF2A mutations led to nonfunctional KIF2A protein (Poirier et al., 2013).

Our generated kif2a knock-out model possessed several similarities to KIF2A mouse models that recapitulated many features of the human phenotype. In particular, $k_{i f 2 a^{-1-}}$ zebrafish larvae had reduced head size compared with their $k i f 2 a^{+/+}$siblings. This neuroanatomical anomaly was supported by neuronal loss in the brain because of a decrease in cell proliferation and increased levels of apoptotic cells in the brains of kif2a $a^{-1-}$ mutants. Correspondingly, Gilet et al. (2020) found that abnormal neuronal migration, large-scale apoptosis, and increased p53 levels during early stages in the entire mouse cortex were likely responsible for the observed microcephaly in KIF2A $A^{+/ H 321 D}$ mice. Programmed cell death was shown to be increased in many mouse models of microcephaly (Poulton et al., 2011; Little and Dwyer, 2019), and, in contrast, a reduction of apoptosis leads to increased brain size (Kuida et al., 1998). Additionally, previous studies have already reported the correlation between KIF2A inactivation and induced apoptosis in cancer cells (Zhang et al., 2016, 2019; Li et al., 2019). The silencing of KIF2A by siRNA resulted in a decrease of PI3 kinase/Akt expression and downstream proteins, a key signaling pathway involved in the regulation of cell proliferation and growth, and ultimately in the initiation of apoptosis (Wang et al., 2014; Zhang et al., 2020). In addition, the neuroprotective role of the PI3 kinase/Akt signaling pathway in zebrafish has already been elucidated, emphasizing its function in the attainment of normal brain size during zebrafish embryogenesis (Chen et al., 2017).

Moreover, Kif2a-cKO mice (Homma et al., 2018) and KIF2A $A^{+/ H 321 D}$ mice (Gilet et al., 2020) presented with behavioral deficiencies and susceptibility to epilepsy. Similarly, kif2a $^{-/-}$larvae showed habituation learning deficits as they were not able to respond to the trained stimulus during the learning period, suggesting inefficient storage of information and memory capacity. These data are in line with memory decay and learning problems, hallmarks of developmental delay and intellectual disability observed in human subjects presenting with KIF2A mutations (Poirier et al., 2013; Tian et al., 2016; Cavallin et al., 2017). We believe that defects in neuronal cell proliferation and increased apoptosis in kif2a ${ }^{-1-}$ mutants may have direct effects on key structures in the brain playing a crucial role in memory. Furthermore, DRE was one of the disease symptoms among patients with mutations in KIF2A (Poirier et al., 2013; Tian et al., 2016; Cavallin et al., 2017). In contrast to the spontaneous epileptiform events observed in Kif2a-cKO mice during postnatal week 5 (Homma et al., 2018), kif2a mutant larvae only exhibited epileptiform events when incubated with a subthreshold dose of a convulsant, PTZ. These findings were supported by the study from Gilet et al. (2020), where KIF2A $A^{+/ H 321 D}$ mice also presented seizure susceptibility after PTZ administration.

Albeit our kif2a zebrafish model recapitulated many phenotypic hallmarks identified in mouse models and human patients with KIF2A mutations, neuroanatomical brain abnormalities described in Kif2a mice models (Homma et al., 2003; Gilet et al., 2020) could not be addressed in our model. This could possibly be because of the fact that the brain ventricular system in mammals is different from that of teleosts (Korzh, 2018), and, although that dorsal pallium likely contains structures homologous to the mammalian hippocampus (Cheng et al., 2014; Saleem and Kannan, 2018), a precise functional mapping of these brain territories is still largely missing. Also, in contrast to the hyperactivity documented in KIF2A mouse models (Homma et al., 2018; Gilet et al., 2020), we observed a reduced locomotor activity in $\mathrm{kif}^{2} \mathrm{a}^{-1-}$ larvae. Although epilepsy is often linked to increased locomotor activity and thus hyperexcitability, various genetic epilepsy zebrafish models have already reported hypoactivity (Scheldeman et al., 2017; Swaminathan et al., 2018; Prentzell et al., 2021). More particularly, some genetic epilepsy mouse models showed hypoactivity as well (Gu et al., 2019), whereas patients are often hyperactive (Pelc et al., 2008). Although light-dark transitions mainly result in hyperactivity (Basnet et al., 2019) because of a boost in the stress or anxiety levels in zebrafish larvae, locomotor behavior strongly depends on precise CNS development and proper functioning of the brain pathways. Our results show that a failure of any of these factors may be responsible for the observed decrease in locomotor activity (Bilotta, 2000; MacPhail et al., 2009; Vignet et al., 2013; Basnet et al., 2019).

Given the fact that Kif2a KO mice died within a day of birth (Homma et al., 2003) and Kif2a-cKO mice died by 
the end of week 6 (Homma et al., 2018), and because kif2a was selectively expressed in the developing brain during embryogenesis, we hypothesized that our (maternal zygotic) kif2a KO zebrafish larvae would die prematurely. Interestingly, kif2a ${ }^{-1-}$ zebrafish larvae survived until adulthood and were fertile, and thus its offspring, hence excluding that survival was essentially regulated by compensatory effects of maternal kif2a transcripts or Kif2a protein. Possibly, some genetic compensation mechanisms could be involved; however, we did not observe any upregulation originating from a direct kinesin-13 family member. We therefore speculate that additional adaptive compensation by other genes may apply; however, such investigations would require the use of transcriptomics, which could provide an elaborate gene ontology enrichment map of upregulated or downregulated genes. Nonetheless, it is also possible that Kif2a function in zebrafish is less crucial than in mice and not necessarily compensated per se. In addition, we observed that kif2a $a^{-1-}$ zebrafish obtained from heterozygous parents had no behavioral phenotype compared with $\mathrm{kif2a}^{-1-}$ zebrafish derived from homozygous fish. Given the substantial role of maternal mRNA in zebrafish oocytes, maternal kif2a might continue to perform its function in early larval development (even after zygotic gene expression initiation), leaving $\mathrm{kif2a}^{-1-}$ larvae from heterozygous parents unaffected (Pelegri, 2003).

Together, we demonstrated that our $\mathrm{kif2a}^{-/-}$zebrafish model mimicked well the important aspects of the phenotype observed in mice models being reminiscent of the phenotype in patients with KIF2A mutations. Our results highlight the value of our novel kif2a $\mathrm{KO}$ model in understanding of the pathologic consequences of KIF2A loss of function. Importantly, since our model does not require tedious genotyping procedures, it could possibly be deployed in medium- to high-throughput MCD/DRE-relevant drug screens and hence used as a high-throughput screening model in a future search for novel and more effective therapies.

\section{References}

Afrikanova T, Serruys AS, Buenafe OE, Clinckers R, Smolders I, de Witte PA, Crawford AD, Esguerra CV (2013) Validation of the zebrafish pentylenetetrazol seizure model: locomotor versus electrographic responses to antiepileptic drugs. PLoS One 8:e54166.

Ali I, Yang WC (2020) The functions of kinesin and kinesin-related proteins in eukaryotes. Cell Adh Migr 14:139-152.

Bahi-Buisson N, Cavallin M (2016) Tubulinopathies overview. In: GeneReviews (, Adam MP, Ardinger HH, Pagon RA, Wallace SE, Bean LJH, Mirzaa G, Amemiya A, eds). Seattle, WA: University of Washington; 1993-2017.

Basnet RM, Zizioli D, Taweedet S, Finazzi D, Memo M (2019) Zebrafish larvae as a behavioral model in neuropharmacology. Biomedicines 7:23.

Bilotta J (2000) Effects of abnormal lighting on the development of zebrafish visual behavior. Behav Brain Res 116:81-87.

Broix L, Asselin L, Silva CG, Ivanova EL, Tilly P, Gilet JG, Lebrun N, Jagline H, Muraca G, Saillour Y, Drouot N, Reilly ML, Francis F, Benmerah A, Bahi-Buisson N, Belvindrah R, Nguyen L, Godin JD, Chelly J, Hinckelmann M-V (2018) Ciliogenesis and cell cycle alterations contribute to KIF2A-related malformations of cortical development. Hum Mol Genet 27:224-238.

Buchsbaum IY, Cappello S (2019) Neuronal migration in the CNS during development and disease: insights from in vivo and in vitro models. Development 146:dev163766.

Cavallin M, Bijlsma EK, El Morjani A, Moutton S, Peeters EA, Maillard C, Pedespan JM, Guerrot AM, Drouin-Garaud V, Coubes C, Genevieve D, Bole-Feysot C, Fourrage C, Steffann J, BahiBuisson N (2017) Recurrent KIF2A mutations are responsible for classic lissencephaly. Neurogenetics 18:73-79.

Chen S, Liu Y, Rong X, Li Y, Zhou J, Lu L (2017) Neuroprotective role of the PI3 kinase/Akt signaling pathway in zebrafish. Front Endocrinol (Lausanne) 8:21.

Cheng RK, Jesuthasan SJ, Penney TB (2014) Zebrafish forebrain and temporal conditioning. Philos Trans R Soc Lond B Biol Sci 369:20120462.

Desikan RS, Barkovich AJ (2016) Malformations of cortical development. Ann Neurol 80:797-810.

Diaz AL, Gleeson JG (2009) The molecular and genetic mechanisms of neocortex development. Clin Perinatol 36:503-512.

Gilet JG, Ivanova EL, Trofimova D, Rudolf G, Meziane H, Broix L, Drouot N, Courraud J, Skory V, Voulleminot P, Osipenko M, BahiBuisson N, Yalcin B, Birling MC, Hinckelmann MV, Kwok BH, Allingham JS, Chelly J (2020) Conditional switching of KIF2A mutation provides new insights into cortical malformation pathogeny. Hum Mol Genet 29:766-784.

Gu B, Zhu M, Glass MR, Rougié M, Nikolova VD, Moy SS, Carney PR, Philpot BD (2019) Cannabidiol attenuates seizures and EEG abnormalities in Angelman syndrome model mice. J Clin Invest 129:5462-5467.

Guerrini R, Dobyns WB (2014) Malformations of cortical development: clinical features and genetic causes. Lancet Neurol 13:710726.

Homma N, Takei Y, Tanaka Y, Nakata T, Terada S, Kikkawa M, Noda Y, Hirokawa N (2003) Kinesin superfamily protein 2A (KIF2A) functions in suppression of collateral branch extension. Cell 114:229239.

Homma N, Zhou R, Naseer MI, Chaudhary AG, Al-Qahtani MH, Hirokawa N (2018) KIF2A regulates the development of dentate granule cells and postnatal hippocampal wiring. Elife 7:e30935.

Hruscha A, Krawitz P, Rechenberg A, Heinrich V, Hecht J, Haass C, Schmid B (2013) Efficient CRISPR/Cas9 genome editing with low off-target effects in zebrafish. Development 140:4982-4987.

Hwang WY, Fu Y, Reyon D, Maeder ML, Tsai SQ, Sander JD, Peterson RT, Yeh JR, Joung JK (2013) Efficient genome editing in zebrafish using a CRISPR-Cas system. Nat Biotechnol 31:227229.

Kalueff AV, Stewart AM, Gerlai R (2014) Zebrafish as an emerging model for studying complex brain disorders. Trends Pharmacol Sci 35:63-75.

Khan KM, Collier AD, Meshalkina DA, Kysil EV, Khatsko SL, Kolesnikova T, Morzherin YY, Warnick JE, Kalueff AV, Echevarria DJ (2017) Zebrafish models in neuropsychopharmacology and CNS drug discovery. Br J Pharmacol 174:1925-1944.

Korzh V (2018) Development of brain ventricular system. Cell Mol Life Sci 75:375-383.

Kuida K, Haydar TF, Kuan CY, Gu Y, Taya C, Karasuyama H, Su MS, Rakic P, Flavell RA (1998) Reduced apoptosis and cytochrome cmediated caspase activation in mice lacking caspase 9. Cell 94:325-337.

Leventer RJ, Phelan EM, Coleman LT, Kean MJ, Jackson GD, Harvey AS (1999) Clinical and imaging features of cortical malformations in childhood. Neurology 53:715-715.

Leventer RJ, Guerrini R, Dobyns WB (2008) Malformations of cortical development and epilepsy. Dialogues Clin Neurosci 10:47-62.

Li X, Shu K, Wang Z, Ding D (2019) Prognostic significance of KIF2A and KIF20A expression in human cancer: a systematic review and meta-analysis. Medicine (Baltimore) 98:e18040. 
Little JN, Dwyer ND (2019) p53 deletion rescues lethal microcephaly in a mouse model with neural stem cell abscission defects. Hum Mol Genet 28:434-447.

MacPhail RC, Brooks J, Hunter DL, Padnos B, Irons TD, Padilla S (2009) Locomotion in larval zebrafish: influence of time of day, lighting and ethanol. Neurotoxicology 30:52-58.

Maor-Nof M, Homma N, Raanan C, Nof A, Hirokawa N, Yaron A (2013) Axonal pruning is actively regulated by the microtubule-destabilizing protein kinesin superfamily protein 2 A. Cell Rep 3:971977.

Miki H, Okada Y, Hirokawa N (2005) Analysis of the kinesin superfamily: insights into structure and function. Trends Cell Biol 15:467-476.

Mueller T, Wullimann M (2016) Atlas of early zebrafish brain development, Ed 2. London: Elsevier.

Nelson JC, Witze E, Ma Z, Ciocco F, Frerotte A, Randlett O, Foskett JK, Granato M (2020) Acute regulation of habituation learning via posttranslational palmitoylation. Curr Biol 30:2729-2738.e4.

Pang T, Atefy R, Sheen V (2008) Malformations of cortical development. Neurologist 14:181-191.

Papayannis CE, Consalvo D, Kauffman MA, Seifer G, Oddo S, D'Alessio L, Saidon P, Kochen S (2012) Malformations of cortical development and epilepsy in adult patients. Seizure 21:377-384.

Pelc K, Cheron G, Dan B (2008) Behavior and neuropsychiatric manifestations in Angelman syndrome. Neuropsychiatr Dis Treat 4:577-584.

Pelegri F (2003) Maternal factors in zebrafish development. Dev Dyn 228:535-554.

Poirier K, Lebrun N, Broix L, Tian G, Saillour Y, Boscheron C, Parrini $E$, Valence $S$, Pierre BS, Oger $M$, Lacombe $D$, Geneviève $D$, Fontana E, Darra F, Cances C, Barth M, Bonneau D, Bernadina BD, N'guyen S, Gitiaux C, et al. (2013) Mutations in TUBG1, DYNC1H1, KIF5C and KIF2A cause malformations of cortical development and microcephaly. Nat Genet 45:639-647.

Poulton CJ, Schot R, Kia SK, Jones M, Verheijen FW, Venselaar H, de Wit MC, de Graaff E, Bertoli-Avella AM, Mancini GM (2011) Microcephaly with simplified gyration, epilepsy, and infantile diabetes linked to inappropriate apoptosis of neural progenitors. Am J Hum Genet 89:265-276.

Prentzell MT, Rehbein U, Cadena Sandoval M, De Meulemeester AS, Baumeister R, Brohée L, Berdel B, Bockwoldt M, Carroll B, Chowdhury SR, von Deimling A, Demetriades C, Figlia G, de Araujo MEG, Heberle AM, Heiland I, Holzwarth B, Huber LA, Jaworski J, Kedra M, et al. (2021) G3BPs tether the TSC complex to lysosomes and suppress mTORC1 signaling. Cell 184:655-674. e27.

Represa A (2019) Why malformations of cortical development cause epilepsy. Front Neurosci 13:250-250.

Saleem S, Kannan RR (2018) Zebrafish: an emerging real-time model system to study Alzheimer's disease and neurospecific drug discovery. Cell Death Discov 4:45.
Scheldeman C, Mills JD, Siekierska A, Serra I, Copmans D, Iyer AM, Whalley BJ, Maes J, Jansen AC, Lagae L, Aronica E, de Witte PAM (2017) mTOR-related neuropathology in mutant tsc2 zebrafish: phenotypic, transcriptomic and pharmacological analysis. Neurobiol Dis 108:225-237.

Siekierska A, Stamberger H, Deconinck T, Oprescu SN, Partoens M, Zhang $Y$, Sourbron J, Adriaenssens E, Mullen P, Wiencek P, Hardies K, Lee J-S, Giong H-K, Distelmaier F, Elpeleg O, Helbig KL, Hersh J, Isikay S, Jordan E, Karaca E, et al. (2019) Biallelic VARS variants cause developmental encephalopathy with microcephaly that is recapitulated in var knockout zebrafish. Nat Commun 10:708.

Stewart AM, Gerlai R, Kalueff AV (2015) Developing highER-throughput zebrafish screens for in-vivo CNS drug discovery. Front Behav Neurosci 9:14.

Sun D, Zhou X, Yu HL, He XX, Guo WX, Xiong WC, Zhu XJ (2017) Regulation of neural stem cell proliferation and differentiation by Kinesin family member 2a. PLoS One 12:e0179047.

Swaminathan A, Hassan-Abdi R, Renault S, Siekierska A, Riché R, Liao M, de Witte PAM, Yanicostas C, Soussi-Yanicostas N, Drapeau P, Samarut É (2018) Non-canonical mTOR-independent role of DEPDC5 in regulating GABAergic network development. Curr Biol 28:1924-1937.e5.

Tian G, Cristancho AG, Dubbs HA, Liu GT, Cowan NJ, Goldberg EM (2016) A patient with lissencephaly, developmental delay, and infantile spasms, due to de novo heterozygous mutation of KIF2A. Mol Genet Genomic Med 4:599-603.

Tiraboschi E, Martina S, van der Ent W, Grzyb K, Gawel K, CorderoMaldonado ML, Poovathingal SK, Heintz S, Satheesh SV, Brattespe J, Xu J, Suster M, Skupin A, Esguerra CV (2020) New insights into the early mechanisms of epileptogenesis in a zebrafish model of Dravet syndrome. Epilepsia 61:549-560.

Vignet C, Bégout ML, Péan S, Lyphout L, Leguay D, Cousin X (2013) Systematic screening of behavioral responses in two zebrafish strains. Zebrafish 10:365-375.

Wang K, Lin C, Wang C, Shao Q, Gao W, Song B, Wang L, Song X, Qu X, Wei F (2014) Silencing Kif2a induces apoptosis in squamous cell carcinoma of the oral tongue through inhibition of the PI3K/Akt signaling pathway. Mol Med Rep 9:273-278.

Wolman MA, Jain RA, Liss L, Granato M (2011) Chemical modulation of memory formation in larval zebrafish. Proc Natl Acad Sci U S A 108:15468-15473.

Zhang Q, Lu D, Liu W, Ye S, Guo H, Liao T, Chen C (2019) Effects of KIF2A on the prognosis of nasopharyngeal carcinoma and nasopharyngeal carcinoma cells. Oncol Lett 18:2718-2723.

Zhang X, Ma C, Wang Q, Liu J, Tian M, Yuan Y, Li X, Qu X (2016) Role of KIF2A in the progression and metastasis of human glioma. Mol Med Rep 13:1781-1787.

Zhang X, Wang Y, Liu X, Zhao A, Yang Z, Kong F, Sun L, Yu Y, Jiang $L$ (2020) KIF2A promotes the progression via AKT signaling pathway and is upregulated by transcription factor ETV4 in human gastric cancer. Biomed Pharmacother 125:109840. 